\title{
41. RELATIVE ABUNDANCES AND RANGES OF SELECT DIATOMS AND SILICOFLAGELLATES FROM SITES 699 AND 704, SUBANTARCTIC SOUTH ATLANTIC ${ }^{1}$
}

\author{
Paul F. Ciesielski²
}

\begin{abstract}
The stratigraphic ranges and relative abundances of selected diatoms and silicoflagellates are presented from three Neogene sedimentary sequences from the subantarctic South Atlantic. These data were compiled from Hole $699 \mathrm{~A}$ in the southwest South Atlantic and Holes 704A and 704B in the southeast South Atlantic. Thirty-five samples were examined from a $67.5-\mathrm{m}$ section of Hole $699 \mathrm{~A}$, which is mostly late Miocene or younger in age. A total of 225 samples was examined from the upper 569.1-m lower Miocene to Quaternary section in Holes 704A and 704B. Although the partial census of the Site 704 sequences is only preliminary, it reveals that the Neogene is remarkably complete and serves as a reference for further detailed examination of an important biostratigraphic-magnetostratigraphic reference section for the Neogene record of the Southern Ocean.
\end{abstract}

\section{INTRODUCTION}

Leg 114 of the Ocean Drilling Program (ODP) drilled several sites in the subantarctic South Atlantic which contain well-preserved Neogene diatoms and silicoflagellates. This data report presents the ranges and relative abundances of selected species of diatoms and silicoflagellates from Hole $699 \mathrm{~A}$ on the lower northeastern slope of the Northeast Georgia Rise $\left(51^{\circ} 32.537^{\prime} \mathrm{S}, 30^{\circ} 40.619^{\prime} \mathrm{W} ; 3705 \mathrm{~m}\right.$ water depth) and Holes 704A and 704B on the Meteor Rise $\left(46^{\circ} 52.76^{\prime} \mathrm{S}\right.$, $7^{\circ} 25.25^{\prime} \mathrm{E} ; 2543 \mathrm{~m}$ water depth) (Fig. 1).

Of the two studied sites, sediments recovered from Site 704 represent the most complete biosiliceous section of the Neogene yet recovered from the Southern Ocean. Detectable hiatuses are only present in the middle Miocene, an interval recovered elsewhere at other Southern Ocean sites. Of particular importance is the presence of a thick lower Miocene section $(\sim 80 \mathrm{~m})$, the first complete and expanded section $(\sim 200 \mathrm{~m})$ of the upper Miocene, and a $187-\mathrm{m}$ section of the upper Pliocene to Quaternary. Many of the studies presented in this volume complement the future use of this site as an important reference section for high-latitude micropaleontology of the Neogene, including detailed benthic, planktonic, and whole-fraction analyses of oxygen and carbon isotopes of the upper Miocene-Quaternary (Hodell and Ciesielski; Müller et al.; Mead et al.), a record of ice-rafted detritus (Allen and Warnke), carbonate and biogenic opal variability (Froelich et al.), and more. The presence of carbonate and biogenic silica throughout the Neogene of Holes 704A and 704B offers an opportunity to cross-calibrate siliceous and calcareous microfossil stratigraphy and correlate with the paleomagnetic record presented by Hailwood and Clement (this volume) and the stable isotopic stratigraphy defined by those previously mentioned.

The Neogene record of Hole $699 \mathrm{~A}$ is less complete than that found at Site 704. The ranges and abundances are recorded herein for the upper Miocene-Quaternary. Significant reworking of siliceous microfossils in the sediments of Hole 699A complicates the stratigraphy of this section and

\footnotetext{
1 Ciesielski, P. F., Kristoffersen, Y., et al., 1991. Proc. ODP, Sci. Results, 114: College Station, TX (Ocean Drilling Program).

2 Department of Geology, University of Florida, Gainesville, FL 32611.
}

may reduce the potential use of it for calibration of species ranges to the paleomagnetic record as defined by Hailwood and Clement (this volume).

It is the author's experience that the initial and last occurrences of species ranges are difficult to accurately define in Southern Ocean sediments because the abundances commonly do not exceed the background level of reworking. In addition, apparent biogeographic variations in their ranges (e.g., Fenner, this volume) complicate their regional applicability. For this reason, various ongoing investigations of recently recovered ODP sequences from the Southern Ocean are concentrating on quantitative studies of species abundances to offer more reliable stratigraphic boundaries based upon major abundance changes within or at the extremes of species ranges. This data report is an attempt to document the relative abundances and ranges of a number of taxa throughout most of the Neogene. This record now serves as a guide for ongoing (P. F. Ciesielski and A. Vrba, unpubl. data) and future quantitative studies of diatom and silicoflagellate occurrences. Some of the results of these quantitative studies are presented in Fenner (this volume). At this time few changes are offered to the Southern Ocean diatom zonation of Weaver and Gombos (1981), Gombos and Ciesielski (1983), and (Ciesielski, 1983), although a much higher resolution stratigraphy calibrated to the magnetostratigraphy and partially based on quantitative studies could be presented at this time. This approach is taken to allow further detailed studies by this and other authors to be completed so as to produce a more permanent stratigraphic zonation. The only change to the existing zonation as employed here is that the lowermost Miocene portion of the Rocella gelida Zone of Gombos and Ciesielski (1983) is replaced by the Rossiella symmetrica Zone, which extends from the last abundant appearance of $R$. gelida to the last consistent $R$. symmetrica as defined by quantitative studies by P. F. Ciesielski and A. Vrba (unpubl. data). Occurrences for these datums at the Leg 114 sites were documented by P. F. Ciesielski (unpubl. data).

\section{PREPARATION OF SAMPLES AND METHODS OF STUDY}

All samples used in this study were collected by the author during Leg 114. All samples were processed for shorebased investigations using the following technique: raw samples were placed in $200-\mathrm{mL}$ beakers and heated with diluted 


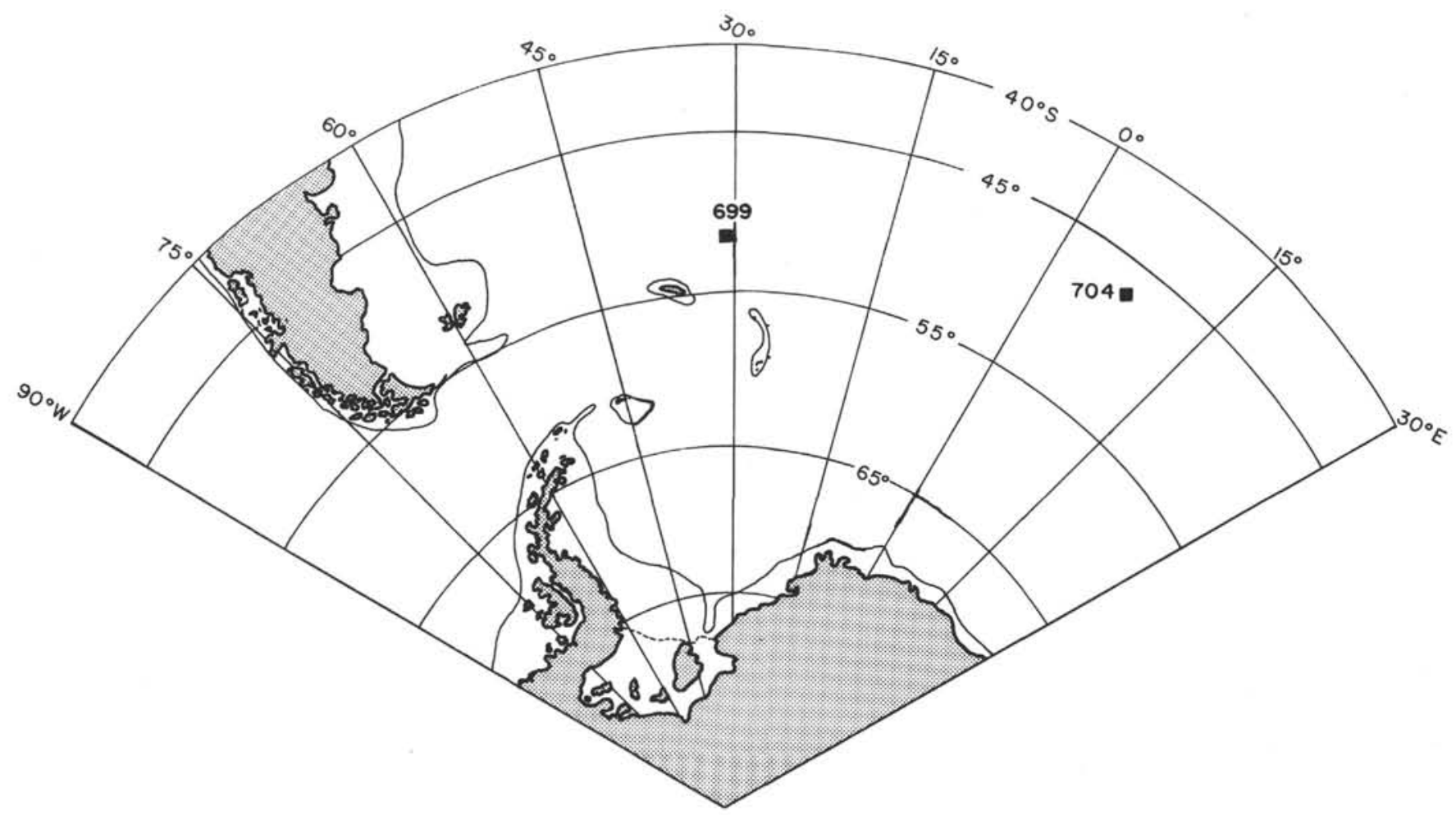

Figure 1. Map of the southern South Atlantic showing the location of Sites 699 and 704.

hydrogen peroxide to disassociate the sediment and remove organic carbon. Hydrochloric acid was then added to dissolve any carbonate present in the samples. The undissolved residues were diluted with distilled water, centrifuged, and decanted to remove the acid. This procedure was repeated three times. Next, the samples were washed with sodium pyrophosphate, centrifuged, and decanted to remove a significant proportion of the clay present in the samples. This step was repeated until the sediment suspension obtained a neutral $\mathrm{pH}$. Processed residues were diluted with distilled water and stored in $50-\mathrm{mL}$ bottles.

Strewn slides of all samples were prepared by shaking bottles containing the sediment and water until all sediment was in suspension, and a small amount of the suspension was pipetted from the middle of the bottle. A few drops of the pipetted solution were placed on a glass slide and dispersed uniformly. After the slides dried, cover slips were mounted using Hyrax (n.d. = 1.71) as the mounting medium.

Relative abundances of selected diatom and silicoflagellate species were determined from Holes 699A (Core 114-699A$1 \mathrm{H}$ through Section 114-699A-8H-2), 704A (entire hole), and 704B (Samples 114-704B-24X-1, 80-82 cm, through 114-704B$62 \mathrm{X}-2,40-42 \mathrm{~cm})$. Relative abundances were recorded as follows:

dominant $=$ more than one specimen/field of view;

abundant $=$ an average of one specimen/field of view;

common = one specimen/five fields of view;

frequent $=$ one specimen $/ 10$ fields of view;

sparse $=$ one specimen $/ 20$ fields of view;

rare $=$ several specimens/slide;

very rare = only one observed specimen.

A "?" designates questionably present specimens, represented by fragments difficult to identify or poorly preserved. Specimens that are interpreted as reworked or displaced are indicated by a lowercase letter in the tables.

\section{RESULTS}

The stratigraphic ranges and relative abundances of selected diatoms and silicoflagellates are given in Table 1 (Hole 699A) and Tables 3 and 4 (Holes 704A and 704B). Accompanying each table is a species locator index citing the column number of alphabetically listed species. Silicoflagellate taxa are indicated by "(S)" after the species name. Paleomagnetic boundaries and chron/subchron identifications for Holes 704A and 704B (Hailwood and Clement, this volume; P. F. Ciesielski, unpubl. data) are presented in Table 2. Similar information was identified for Hole 699A (P. F. Ciesielski, unpubl. data).

References relating to the taxonomy of the cited taxa are not provided in this data report; however, they are readily available in widely accessible literature. Taxa endemic to the Southern Ocean are referenced and/or described and figured by the following: Schrader (1976), Fenner et al. (1976), Gombos (1977), Weaver and Gombos (1981), Gombos and Ciesielski (1983), Ciesielski (1983, 1985), and Fenner (this volume). Additional references to Southern Ocean diatom taxa and more cosmopolitan species are given in Barron (1981, 1983, 1985a) and Barron and Bauldauf (1986). The reader is referred to several other references for the few silicoflagellate taxa listed herein (Busen and Wise, 1977; Shaw and Ciesielski, 1983; Ciesielski et al., 1989; Ciesielski, this volume).

All siliceous and calcareous microfossil datums and paleomagnetic boundaries from Holes 699A, 704A, and 704B were tabulated in a biostratigraphic-magnetostratigraphic synthesis of ODP Leg 114 (P. F. Ciesielski, unpubl. data), which also figures the occurrence of all microfossil zonal schemes in relation to the paleomagnetic record. It is important to note that the data presented here do not represent all the compiled data regarding diatom and silicoflagellate ranges in the discussed holes. Fenner (this volume) presents quantitative data 
of selected species ranges from the upper Pliocene-Quaternary of these holes and P. F. Ciesielski and A. Vrba (unpubl. data) have examined quantitative fluctuations of species from the upper Oligocene-lowermost Miocene section of Hole 699A and other taxa from the lower to lower upper Pliocene of Hole 704A. These results (P. F. Ciesielski, unpubl. data) have been combined to cite the depths, ages, and bracketing samples of Neogene silicoflagellate and diatom datums, in this volume.

\section{ACKNOWLEDGMENTS}

The National Science Foundation and the Joint Oceanographic Institutions, Inc., are thanked for their sponsorship of the shipboard participation of PFC on Ocean Drilling Program Leg 114. Financial support for this purpose was provided through the Texas A\&M Research Foundation/United States Science Program award P.O. 20038. The same institutions are also thanked for their post-cruise financial support through award P.O. 20115. Dr. A. M. Gombos, Jr., is thanked for his critical review of this report. Ms. Susan Case-Ciesielski provided editorial and drafting and computer assistance.

\section{REFERENCES}

Barron, J. A., 1981. Late Cenozoic diatom biostratigraphy and paleoceanography of the middle-latitude eastern North Pacific, Deep Sea Drilling Project Leg 63. In Yeats, R. S., Haq, B. U., et al., Init. Repts. DSDP, 63: Washington (U.S. Govt. Printing Office), 507-535.

1983. Latest Oligocene through early middle Miocene diatom biostratigraphy of the eastern tropical Pacific. Mar. Micropaleontol., 7:487-515.

1985a. Late Eocene to Holocene diatom biostratigraphy of the equatorial Pacific Ocean, Deep Sea Drilling Project Leg 85. In Mayer, L., Theyer, F., et al., Init. Repts. DSDP, 85: Washington (U.S. Govt. Printing Office), 413-457.

1985b. Miocene to Holocene planktic diatoms. In Bolli, H. M., Saunders, J. B., and Perch-Nielsen, K. (Eds.), Plankton Stratigraphy: Cambridge (Cambridge Univ. Press), 763-810.

Barron, J. A., and Baldauf, J. G., 1986. Diatom stratigraphy of the lower Pliocene part of the Sisquoc Formation, Harris Grade section, California. Micropaleontology, 32:357-371.

Bukry, 1981. Synthesis of silicoflagellate stratigraphy for Maestrichtian to Quaternary marine sediments. In Warme, T. E., Douglas, R. C., and Winterer, E. L. (Eds.), The Deep Sea Drilling Project: A Decade of Progress. Soc. Econ. Paleontol. Mineral. Spec. Publ., 32:433-444.

Busen, K. E., and Wise, S. W., Jr., 1977. Silicoflagellate stratigraphy, Deep Sea Drilling Project Leg 36. In Barker, P., Dalziel, I.W.D., et al., Init. Repts. DSDP, 36: Washington (U.S. Govt. Printing Office), 697-743.
Ciesielski, P. F., 1983. The Neogene and Quaternary diatom biostratigraphy of subantarctic sediments, Deep Sea Drilling Project Leg 71. In Ludwig, W. J., Krasheninnikov, V. A., et al., Init. Repts. DSDP, 71 (Pt. 2): Washington (U.S. Govt. Printing Office), 635-666.

1985. Middle Miocene to Quaternary diatom biostratigraphy of Deep Sea Drilling Project Site 594, Chatham Rise, southwest Pacific. In Kennett, J. P., von der Borch, C. C., et al., Init. Repts. DSDP, 90: Washington (U.S. Govt. Printing Office), 863885.

Ciesielski, P. F., Hasson, P., and Turner, J. W., 1989. The stratigraphy of Neogene silicoflagellates from the Norwegian-Greenland Sea, ODP Leg 104. In Eldholm, O., Theide, J., et al., Proc. ODP, Sci. Results, 104: College Station, TX (Ocean Drilling Program), 497-525.

Fenner, J., Schrader, H. J., and Wienigk, H., 1976. Diatom phytoplankton studies in the southern Pacific Ocean, composition and correlation to the Antarctic Convergence and its paleoecological significance. In Hollister, C. D., Craddock, C., et al., Init. Repts. DSDP, 35: Washington (U.S. Govt. Printing Office), 757-813.

Gombos, A. M., 1977. Paleogene and Neogene diatoms from the Falkland Plateau and Malvinas Outer Basin: Leg 36, Deep Sea Drilling Project. In Barker, P., Dalziel, I.W.D., et al., Init. Repts. DSDP, 36: Washington (U.S. Govt. Printing Office), 575-690.

Gombos, A. M., Jr., and Ciesielski, P. F., 1983. Late Eocene to early Miocene diatoms from the southwest Atlantic. In Ludwig, W. J., Krasheninnikov, V. A., et al., Init. Repts. DSDP, 71 (Pt. 2): Washington (U.S. Govt. Printing Office), 583-684.

Martini, E., 1971. Neogene silicoflagellates from the equatorial $\mathrm{Pa}$ cific. In Winterer, E. L., Riedel, W. R., et al., Init. Repts. DSDP, 7 (Pt. 2): Washington (U.S. Govt. Printing Office), 1695-1708. 1972. Silicoflagellate zones in the late Oligocene and early Miocene of Europe. Senckenbergiana Lethaea, 53:119-122.

Schrader, H.-J., 1976. Cenozoic planktonic diatom biostratigraphy of the Southern Pacific Ocean. In Hollister, C. D., Craddock, C., et al., Init. Repts. DSDP, 35: Washington (U.S. Govt. Printing Office), 605-672.

Shaw, C. A., and Ciesielski, P. F., 1983. Silicoflagellate biostratigraphy of middle Eocene to Holocene subantarctic sediments recovered by Deep Sea Drilling Project Leg 71. In Ludwig, W. J., Krasheninnikov, V. A., et al., Init. Repts. DSDP, 71 (Pt. 2): Washington (U.S. Govt. Printing Office), 687-737.

Weaver, F. M., and Gombos, A. M., 1981. Southern high-latitude diatom biostratigraphy. In Warme, T. E., Douglas, R. G., and Winterer, E. L. (Eds.), The Deep Sea Drilling Project: A Decade of Progress: Spec. Publ. Soc. Econ. Paleontol. Mineral., 32:445470.

Date of initial receipt: 5 December 1989

Date of acceptance: 14 December 1989

Ms 114B-138 
Southern Ocean diatom zone (Weaver and Gombos, 1981;

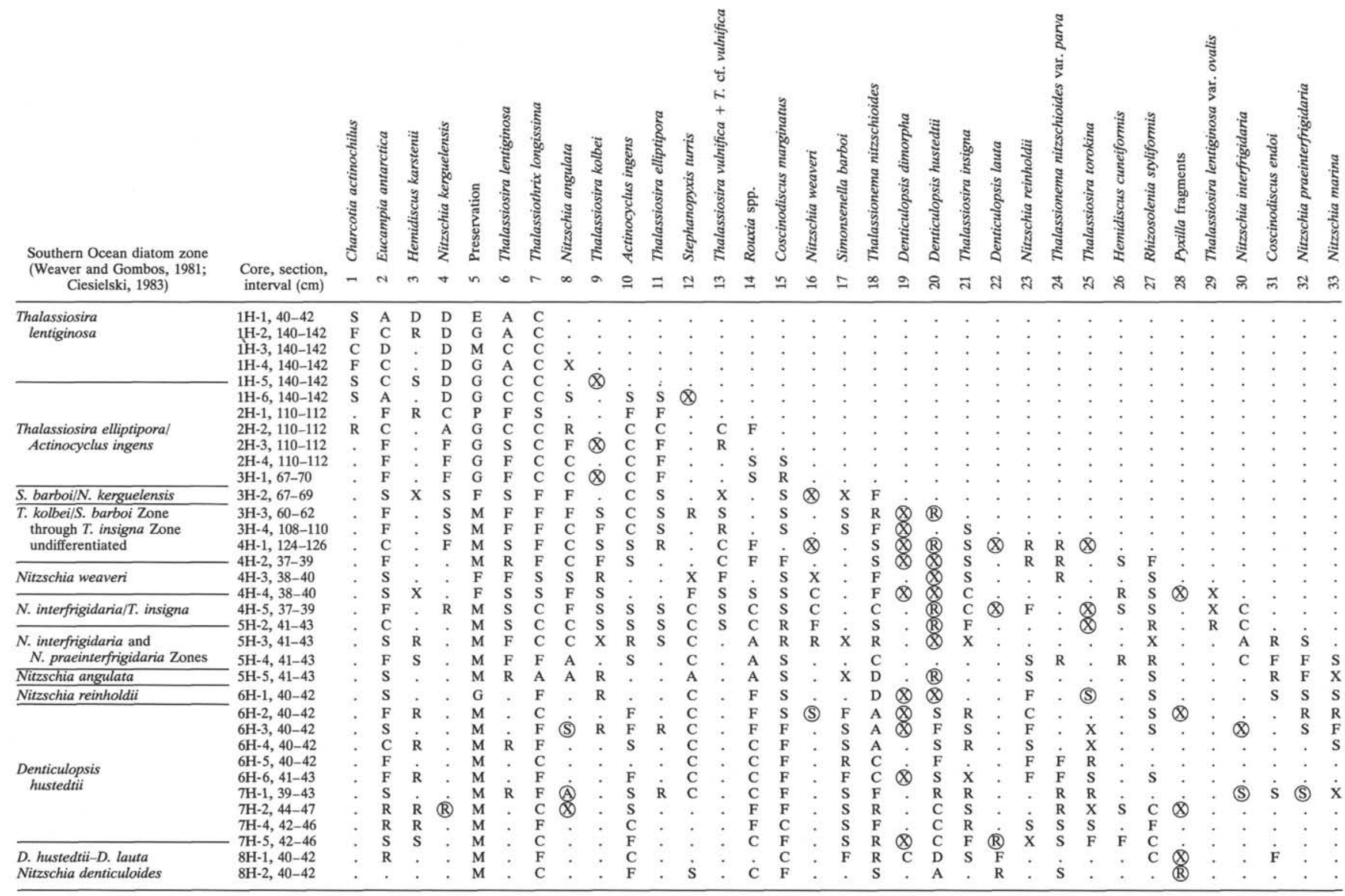

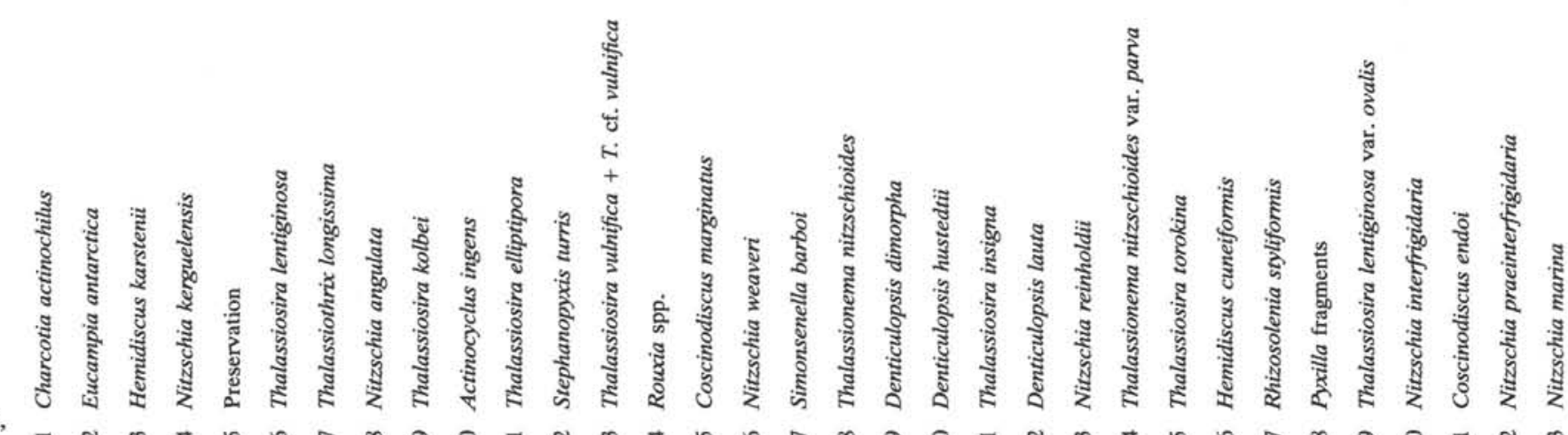




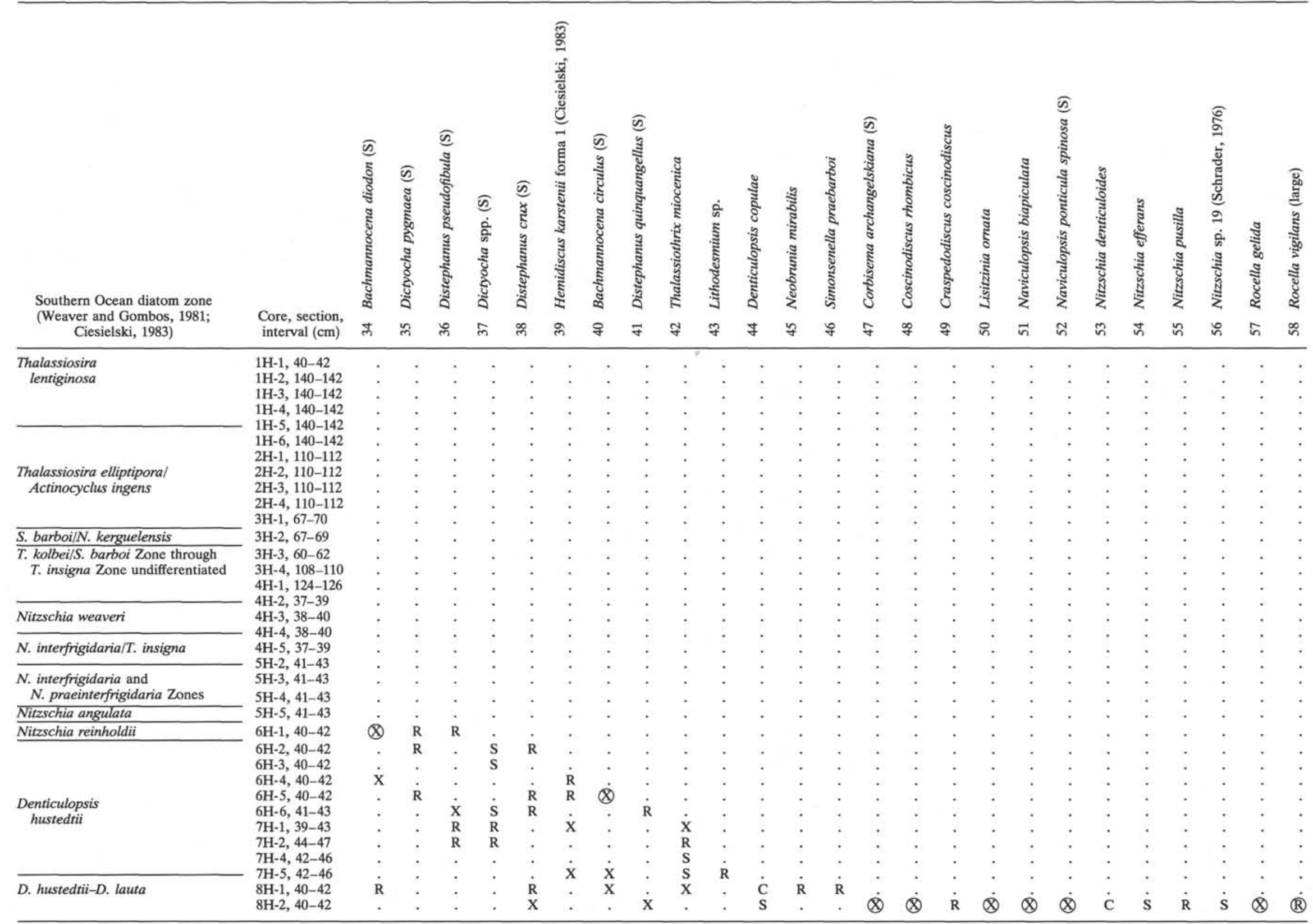

Nitzschia weaveri $\quad 4 \mathrm{H}-3,38-40$ N. interfrigidaria and
$N$. praeinterfrigidaria Zone 40-42 $8 \mathrm{H}-2,40-42$ 
Species location index

Index number is the column in which species appears.

\begin{tabular}{|c|c|}
\hline $\begin{array}{c}\text { Index } \\
\text { number }\end{array}$ & Species \\
\hline 10 & Actinocyclus ingens \\
\hline 40 & Bachmannocena circulus (S) \\
\hline 34 & Bachmannocena diodon (S) \\
\hline 1 & Charcotia actinochilus \\
\hline 47 & Corbisema archangelskiana (S) \\
\hline 31 & Coscinodiscus endoi \\
\hline 9 & Thalassiosira kolbei \\
\hline 15 & Coscinodiscus marginatus \\
\hline 48 & Coscinodiscus rhombicus \\
\hline 49 & Craspedodiscus coscinodiscus \\
\hline 44 & Denticulopsis copulae \\
\hline 19 & Denticulopsis dimorpha \\
\hline 20 & Denticulopsis hustedtii \\
\hline 22 & Denticulopsis lauta \\
\hline 35 & Dictyocha pygmaea (S) \\
\hline 37 & Dictyocha spp. (S) \\
\hline 38 & Distephanus crux (S) \\
\hline 36 & Distephanus pseudofibula (S) \\
\hline 41 & Distephanus quinquangellus \\
\hline 2 & Eucampia antarctica \\
\hline 26 & Hemidiscus cuneiformis \\
\hline 3 & Hemidiscus karstenii \\
\hline 39 & Hemidiscus karstenii forma 1 (Ciesielski, 1983) \\
\hline 50 & Lisitzinia ornata \\
\hline 43 & Lithodesmium sp. \\
\hline 51 & Naviculopsis biapiculata (S) \\
\hline 52 & Naviculopsis ponticula spinosa (S) \\
\hline 45 & Neobrunia mirabilis \\
\hline 8 & Nitzschia angulata \\
\hline 53 & Nitzschia denticuloides \\
\hline 54 & Nitzschia efferans \\
\hline 30 & Nitzschia interfrigidaria \\
\hline 4 & Nitzschia kerguelensis \\
\hline 33 & Nitzschia marina \\
\hline 32 & Nitzschia praeinterfrigidaria \\
\hline 55 & Nitzschia pusilla \\
\hline 23 & Nitzschia reinholdii \\
\hline 56 & Nitzschia sp. 19 (Schrader, 1976) \\
\hline 16 & Nitzschia weaveri \\
\hline 5 & Preservation \\
\hline 28 & Pyxilla fragments \\
\hline 27 & Rhizosolenia styliformis \\
\hline 57 & Rocella gelida \\
\hline 58 & Rocella vigilans (large) \\
\hline 14 & Rouxia spp. \\
\hline 17 & Simonsenella barboi \\
\hline 46 & Simonsenella praebarboi \\
\hline 12 & Stephanopyxis turris \\
\hline 18 & Thalassionema nitzschioides \\
\hline 24 & Thalassionema nitzschioides var. parva \\
\hline 11 & Thalassiosira elliptipora \\
\hline 21 & Thalassiosira insigna \\
\hline 6 & Thalassiosira lentiginosa \\
\hline 29 & Thalassiosira lentiginosa var. ovalis \\
\hline 25 & Thalassiosira torokina \\
\hline 13 & Thalassiosira vulnifica $+T$. cf. vulnifica \\
\hline 7 & Thalassiothrix longissima \\
\hline 42 & Thalassiothrix miocenica \\
\hline
\end{tabular}

Note: $\mathrm{X}=$ very rare $\mathrm{R}=$ rare $\mathrm{S}=$ sparse $\mathrm{F}=$ frequent; $\mathrm{C}=$ common $; \mathrm{A}=$ abundant $\mathrm{D}=$ dominant $\mathrm{P}=$ poor; $\mathrm{F}=$ fair $\mathrm{M}=$ moderate $\mathrm{G}=$ good $\mathrm{E}=$ excellent $;$ ? = questionably present; . = not present. 
Table 2. Paleomagnetic datums in Holes 704A and 704B as defined by Hailwood and Clement (this volume).

\begin{tabular}{|c|c|c|c|c|}
\hline Paleomagnetic datum & $\begin{array}{l}\text { Age } \\
\text { (Ma) }\end{array}$ & Bracketing samples & $\begin{array}{l}\text { Depth range } \\
\text { (mbsf) }\end{array}$ & $\begin{array}{c}\text { Mean } \\
\text { position } \\
\text { (mbsf) }\end{array}$ \\
\hline Brunhes/Matuyama boundary & 0.73 & $704 \mathrm{~B}-4 \mathrm{H}-6,6 \mathrm{~cm} / 5 \mathrm{H}-1,57 \mathrm{~cm}$ & $33.26-35.77$ & 34.51 \\
\hline Top Jaramillo Subchron & 0.91 & $704 \mathrm{~B}-5 \mathrm{H}-2,132 \mathrm{~cm} / 5 \mathrm{H}-3,61 \mathrm{~cm}$ & $38.02-38.81$ & 38.41 \\
\hline Base Jaramillo Subchron & 0.98 & $704 \mathrm{~B}-5 \mathrm{H}-6,100 \mathrm{~cm} / 5 \mathrm{H}-7,16 \mathrm{~cm}$ & $43.70-44.85$ & 44.27 \\
\hline Top Olduvai Subchron & 1.66 & $704 \mathrm{~A}-10 \mathrm{H}-4,100 \mathrm{~cm} / 10 \mathrm{H}-5,31 \mathrm{~cm}$ & $88.69-89.50$ & 89.09 \\
\hline Matuyama/Gauss boundary & 2.47 & $704 \mathrm{~A}-18 \mathrm{X}-6,145 \mathrm{~cm} / 19 \mathrm{X}-1,5 \mathrm{~cm}$ & $168.15-168.75$ & 168.75 \\
\hline Top Kaena Subchron & 2.92 & $704 \mathrm{~A}-19 \mathrm{X}-5,126 \mathrm{~cm} / 19 \mathrm{X}-6,5 \mathrm{~cm}$ & $176.95-176.25$ & 176.10 \\
\hline Base Kaena Subchron & 2.99 & $704 \mathrm{~A}-19 \mathrm{X}-7,5 \mathrm{~cm} / 19 \mathrm{X}-7,15 \mathrm{~cm}$ & $177.75-177.85$ & 177.80 \\
\hline Top Mammoth Subchron & 3.08 & $704 \mathrm{~A}-20 \mathrm{X}-1,85 \mathrm{~cm} / 20 \mathrm{X}-1,95 \mathrm{~cm}$ & $179.05-179.15$ & 179.10 \\
\hline Base Mammoth Subchron & 3.18 & $704 \mathrm{~A}-20 \mathrm{X}-3,15 \mathrm{~cm} / 20 \mathrm{X}-3,45 \mathrm{~cm}$ & $181.35-181.65$ & 181.50 \\
\hline Gauss/Gilbert boundary & 3.40 & $704 \mathrm{~A}-20 \mathrm{X}-6,85 \mathrm{~cm} / 20 \mathrm{X}-6,104 \mathrm{~cm}$ & $186.55-186.75$ & 186.65 \\
\hline Top Cochiti Subchron & 3.88 & $704 \mathrm{~A}-22 \mathrm{X}-1,115 \mathrm{~cm} / 22 \mathrm{X}-2,5 \mathrm{~cm}$ & $198.35-198.75$ & 198.55 \\
\hline Base Cochiti Subchron & 3.97 & $704 \mathrm{~A}-22 \mathrm{X}-4,15 \mathrm{~cm} / 22 \mathrm{X}-4,35 \mathrm{~cm}$ & $201.85-202.05$ & 201.95 \\
\hline Top Nunivak Subchron & 4.10 & $704 \mathrm{~A}-22 \mathrm{X}-5,95 \mathrm{~cm} / 22 \mathrm{X}-5,104 \mathrm{~cm}$ & $204.15-204.24$ & 204.20 \\
\hline Base Nunivak Subchron & 4.24 & $704 \mathrm{~A}-23 \mathrm{X}-3,55 \mathrm{~cm} / 23 \mathrm{X}-3,75 \mathrm{~cm}$ & $210.25-210.45$ & 210.35 \\
\hline Top Sidufjall Subchron & 4.40 & $704 \mathrm{~A}-23 \mathrm{X}-4,75 \mathrm{~cm} / 23 \mathrm{X}-4,85 \mathrm{~cm}$ & $211.95-212.05$ & 212.00 \\
\hline Base Sidufjall Subchron & 4.47 & $704 \mathrm{~A}-23 \mathrm{X}-5,45 \mathrm{~cm} / 23 \mathrm{X}-5,65 \mathrm{~cm}$ & $213.15-213.35$ & 213.25 \\
\hline Top Thvera Subchron & 4.57 & $704 \mathrm{~A}-23 \mathrm{X}-6,10 \mathrm{~cm} / 24 \mathrm{X}-1,33 \mathrm{~cm}$ & $214.29-216.52$ & 215.40 \\
\hline Base Thvera Subchron & 4.77 & $704 \mathrm{~A}-24 \mathrm{X}-3,15 \mathrm{~cm} / 24 \mathrm{X}-3,35 \mathrm{~cm}$ & $219.35-219.55$ & 219.45 \\
\hline Gilbert/C3AN boundary & 5.35 & $704 \mathrm{~B}-25 \mathrm{X}-1,135 \mathrm{~cm} / 25 \mathrm{X}-2,28 \mathrm{~cm}$ & $224.55-224.97$ & 224.76 \\
\hline C3AN.33 & 5.53 & $704 \mathrm{~B}-25 \mathrm{X}-6,25 \mathrm{~cm} / 25 \mathrm{X}-6,45 \mathrm{~cm}$ & $230.95-231.15$ & 231.05 \\
\hline C3AN.61 & 5.68 & $704 \mathrm{~B}-26 \mathrm{X}-1,104 \mathrm{~cm} / 26 \mathrm{X}-1,125 \mathrm{~cm}$ & $233.74-233.95$ & 233.85 \\
\hline C3AN/C3AR boundary & 5.89 & $704 \mathrm{~B}-26 \mathrm{X}-5,45 \mathrm{~cm} / 27 \mathrm{X}-2,41 \mathrm{~cm}$ & $239.14-244.10$ & 241.62 \\
\hline C3AR.59 & 6.37 & $704 \mathrm{~B}-27 \mathrm{X}-6,135 \mathrm{~cm} / 27 \mathrm{X}-7,12 \mathrm{~cm}$ & $251.05-251.45$ & 251.25 \\
\hline C3AR.75 & 6.50 & $704 \mathrm{~B}-28 \mathrm{X}-4,45 \mathrm{~cm} / 28 \mathrm{X}-4,65 \mathrm{~cm}$ & $256.65-256.85$ & 256.75 \\
\hline C3AR/C4N boundary & 6.70 & $704 \mathrm{~B}-28 \mathrm{X}-6,25 \mathrm{~cm} / 28 \mathrm{X}-6,35 \mathrm{~cm}$ & $259.45-259.55$ & 259.50 \\
\hline C4N.1 & 6.78 & $704 \mathrm{~B}-29 \mathrm{X}-2,115 \mathrm{~cm} / 29 \mathrm{X}-3,56 \mathrm{~cm}$ & $263.84-264.75$ & 264.29 \\
\hline C 4 N.2 & 6.85 & $704 \mathrm{~B}-29 \mathrm{X}-3,115 \mathrm{~cm} / 29 \mathrm{X}-4,56 \mathrm{~cm}$ & $265.34-266.25$ & 265.79 \\
\hline C 4 N. 8 & 7.28 & $704 \mathrm{~B}-29 \mathrm{X}-4,115 \mathrm{~cm} / 29 \mathrm{X}-5,56 \mathrm{~cm}$ & $266.84-267.75$ & 267.29 \\
\hline C4N.9 & 7.35 & $704 \mathrm{~B}-29 \mathrm{X}-7,31 \mathrm{~cm} / 30 \mathrm{X}-1,45 \mathrm{~cm}$ & $270.50-271.15$ & 270.82 \\
\hline C4N/C4R boundary & 7.41 & $704 \mathrm{~B}-30 \mathrm{X}-4,55 \mathrm{~cm} / 30 \mathrm{X}-4,75 \mathrm{~cm}$ & $275.75-275.95$ & 275.85 \\
\hline C4R/C4AN boundary & 7.90 & 704B-32X-1, $35 \mathrm{~cm} / 32 \mathrm{X}-1,39 \mathrm{~cm}$ & $290.05-290.08$ & 290.06 \\
\hline C4AN.52 & 8.21 & $704 \mathrm{~B}-35 \mathrm{X}-1,25 \mathrm{~cm} / 35 \mathrm{X}-1,45 \mathrm{~cm}$ & $318.45-318.65$ & 318.55 \\
\hline C4AN.85 & 8.41 & $704 \mathrm{~B}-35 \mathrm{X}-4,125 \mathrm{~cm} / 35 \mathrm{X}-5,5 \mathrm{~cm}$ & $323.95-324.25$ & 324.10 \\
\hline C4AN/C4AR boundary & 8.50 & $704 \mathrm{~B}-35 \mathrm{X}-6,115 \mathrm{~cm} / 35 \mathrm{X}-6,145 \mathrm{~cm}$ & $326.85-327.15$ & 327.05 \\
\hline C4AR.50 & 8.71 & $704 \mathrm{~B}-37 \mathrm{X}-1,61 \mathrm{~cm} / 37 \mathrm{X}-2,61 \mathrm{~cm}$ & $337.80-339.30$ & 338.55 \\
\hline C $5 \mathrm{~N} / \mathrm{CSR}$ boundary & 10.42 & $704 \mathrm{~B}-44 \mathrm{X}-2,54 \mathrm{~cm} / 45 \mathrm{X}-1,36 \mathrm{~cm}$ & $405.73-413.55$ & 409.64 \\
\hline \multirow[t]{2}{*}{${ }^{\mathrm{a}}$ Base C5R (truncated by hiatus) } & $<11.55$ & $704 \mathrm{~B}-46 \mathrm{X}-5,80 \mathrm{~cm} / 46 \mathrm{X}-4,82 \mathrm{~cm}$ & $429.50-428.02$ & 428.76 \\
\hline & Hiatus & $704 \mathrm{~B}-6 \mathrm{X}-5,80 \mathrm{~cm} / 46 \mathrm{X}-4,82 \mathrm{~cm}$ & $429.50-428.02$ & 428.76 \\
\hline${ }^{\mathrm{a}}$ Top C5ACR (truncated by hiatus) & 14.08 & $704 \mathrm{~B}-46 \mathrm{X}-5,80 \mathrm{~cm} / 46 \mathrm{X}-4,82 \mathrm{~cm}$ & $429.50-428.02$ & 428.76 \\
\hline C5ACR/C5ADN boundary & 14.20 & $704 \mathrm{~B}-46 \mathrm{X}-5,95 \mathrm{~cm} / 46 \mathrm{X}-5,85 \mathrm{~cm}$ & $429.65-429.55$ & 429.60 \\
\hline C5ADN/C5ADR boundary & 14.66 & $704 \mathrm{~B}-47 \mathrm{X}-3,45 \mathrm{~cm} / 47 \mathrm{X}-3,55 \mathrm{~cm}$ & $435.65-435.75$ & 435.70 \\
\hline C5ADR/C5BN boundary & 14.87 & $704 \mathrm{~B}-47 \mathrm{X}-3,135 \mathrm{~cm} / 47 \mathrm{X}-4,5 \mathrm{~cm}$ & $436.55-436.75$ & 436.65 \\
\hline C5BN.2 & 14.96 & $704 \mathrm{~B}-47 \mathrm{X}-4,65 \mathrm{~cm} / 47 \mathrm{X}-4,75 \mathrm{~cm}$ & $437.35-437.45$ & 437.40 \\
\hline C5BN.6 & 15.13 & $704 \mathrm{~B}-47 \mathrm{X}-5,115 \mathrm{~cm} / 47 \mathrm{X}-6,40 \mathrm{~cm}$ & $439.35-440.09$ & 439.72 \\
\hline C5BN/C5BR boundary & 15.27 & $704 \mathrm{~B}-48 \mathrm{X}-1,56 \mathrm{~cm} / 48 \mathrm{X}-2,56 \mathrm{~cm}$ & $442.25-443.75$ & 443.00 \\
\hline \multirow{2}{*}{${ }^{\mathrm{a} B a s e}$ C5BR (truncated by hiatus) } & $<16.22$ & $704 \mathrm{~B}-49 \mathrm{X}-1,75 \mathrm{~cm} / 49 \mathrm{X}-1,115 \mathrm{~cm}$ & $451.95-452.35$ & 452.15 \\
\hline & Hiatus & $704 \mathrm{~B}-9 \mathrm{X}-1,75 \mathrm{~cm} / 49 \mathrm{X}-1,115 \mathrm{~cm}$ & $451.95-452.35$ & 452.15 \\
\hline${ }^{\mathrm{a}} \mathrm{Top} \mathrm{C} 5 \mathrm{CN} .8$ (truncated by hiatus) & $>16.80$ & $704 \mathrm{~B}-49 \mathrm{X}-1,75 \mathrm{~cm} / 49 \mathrm{X}-1,115 \mathrm{~cm}$ & $451.95-452.35$ & 452.15 \\
\hline C5CN/C5CR boundary & 16.98 & $704 \mathrm{~B}-49 \mathrm{X}-2,55 \mathrm{~cm} / 49 \mathrm{X}-2,125 \mathrm{~cm}$ & $453.25-453.95$ & 453.60 \\
\hline C5CR/C5DN boundary & 17.57 & $704 \mathrm{~B}-53 \mathrm{X}-3,145 \mathrm{~cm} / 54 \mathrm{X}-2,15 \mathrm{~cm}$ & $486.15-492.85$ & 489.50 \\
\hline C5DN/C5DR boundary & 17.90 & $704 \mathrm{~B}-54 \mathrm{X}-5,95 \mathrm{~cm} / 54 \mathrm{X}-5,104 \mathrm{~cm}$ & $498.15-498.25$ & 498.20 \\
\hline C5EN/C5ER boundary & 19.09 & $704 \mathrm{~B}-56 \mathrm{X}-2,65 \mathrm{~cm} / 56 \mathrm{X}-2,115 \mathrm{~cm}$ & $512.34-512.84$ & 512.59 \\
\hline C5ER/C6N boundary & 19.35 & $704 \mathrm{~B}-56 \mathrm{X}-3,65 \mathrm{~cm} / 56 \mathrm{X}-3,85 \mathrm{~cm}$ & $513.84-514.04$ & 513.94 \\
\hline C6N/C6R boundary & 20.45 & $704 \mathrm{~B}-58 \mathrm{X}-5,55 \mathrm{~cm} / 58 \mathrm{X}-5,65 \mathrm{~cm}$ & $535.74-535.84$ & 535.79 \\
\hline C6R/C6AN boundary & 20.88 & 704B-58X-6, $85 \mathrm{~cm} / 59 \mathrm{X}-1,35 \mathrm{~cm}$ & $537.54-539.04$ & 538.29 \\
\hline C6AAN/C6AAR boundary & 22.35 & $704 \mathrm{~B}-61 \mathrm{X}-1,125 \mathrm{~cm} / 61 \mathrm{X}-1,135 \mathrm{~cm}$ & $558.94-559.04$ & 558.99 \\
\hline C6BN/C6BR boundary & 22.97 & $704 \mathrm{~B}-62 \mathrm{X}-3,35 \mathrm{~cm} / 62 \mathrm{X}-3,55 \mathrm{~cm}$ & $570.54-570.74$ & 570.64 \\
\hline
\end{tabular}

${ }^{\text {a }}$ Paleomagnetic chron not completely represented because of the hiatus that has removed an undetermined portion of it. 


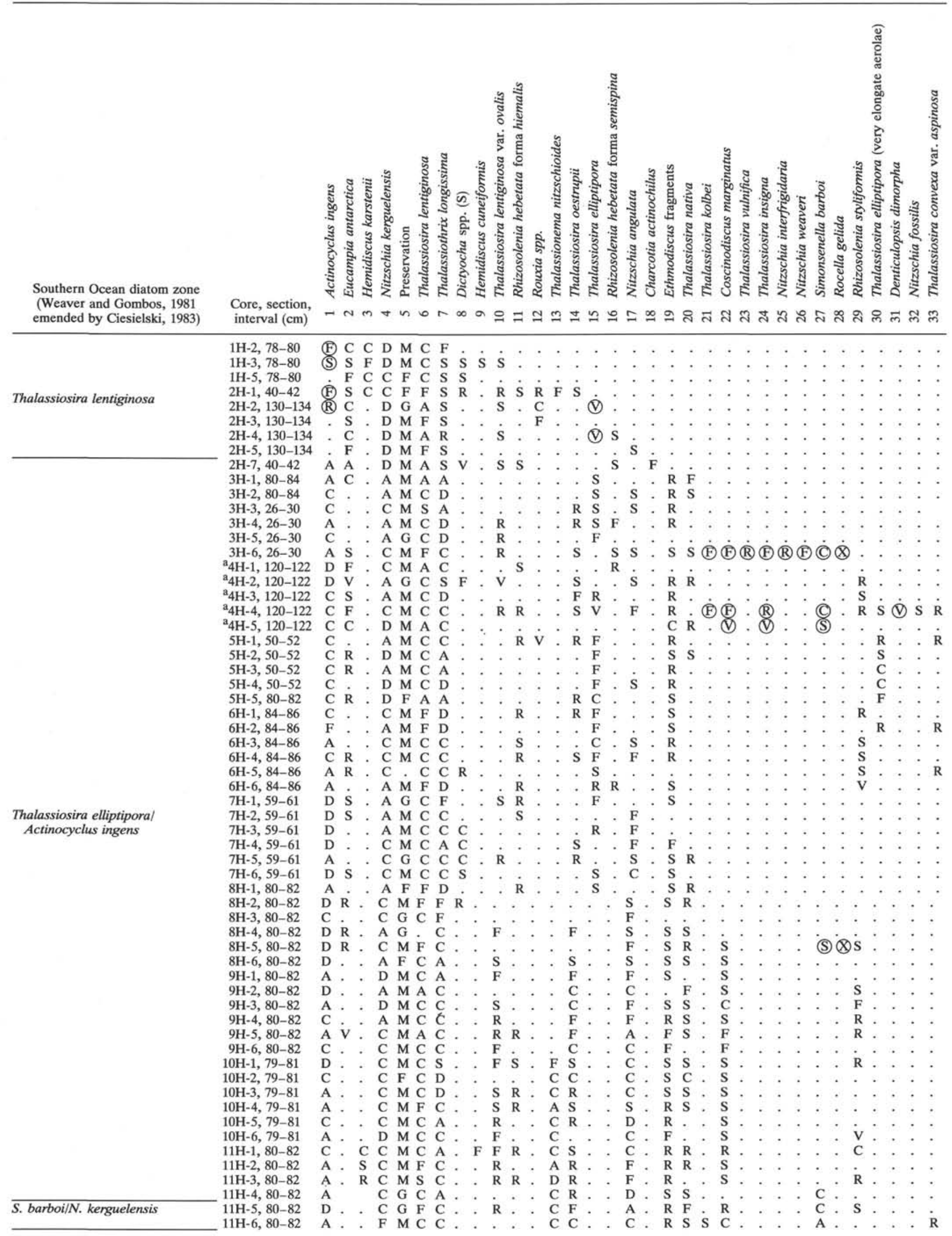


Southern Ocean diatom zone (Weaver and Gombos, 1981 emended by Ciesielski, 1983) Thalassiosira kolbei/
Simonsenella barboi

Thalassiosira vulnifica

Thalassiosira insigna

Nitzschia weaveri and Nitzschia

interfrigidaria/Thalassiosira

vulnifica zonal equivalent

Nitzschia interfrigidaria

Nitzschia angulata

Nitzschia reinholdit
Core, section, interval $(\mathrm{cm})$

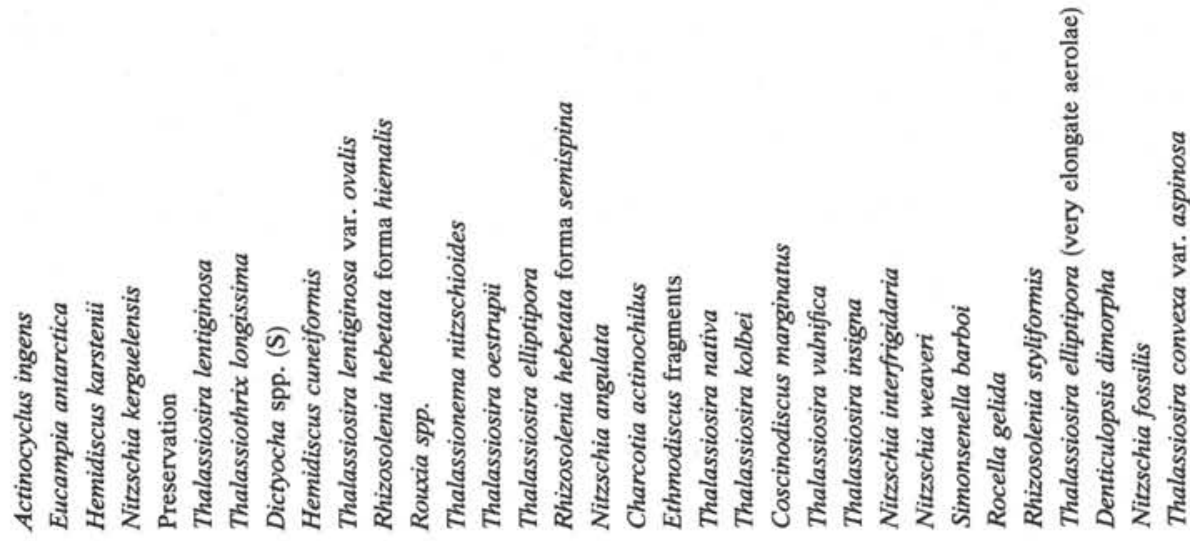

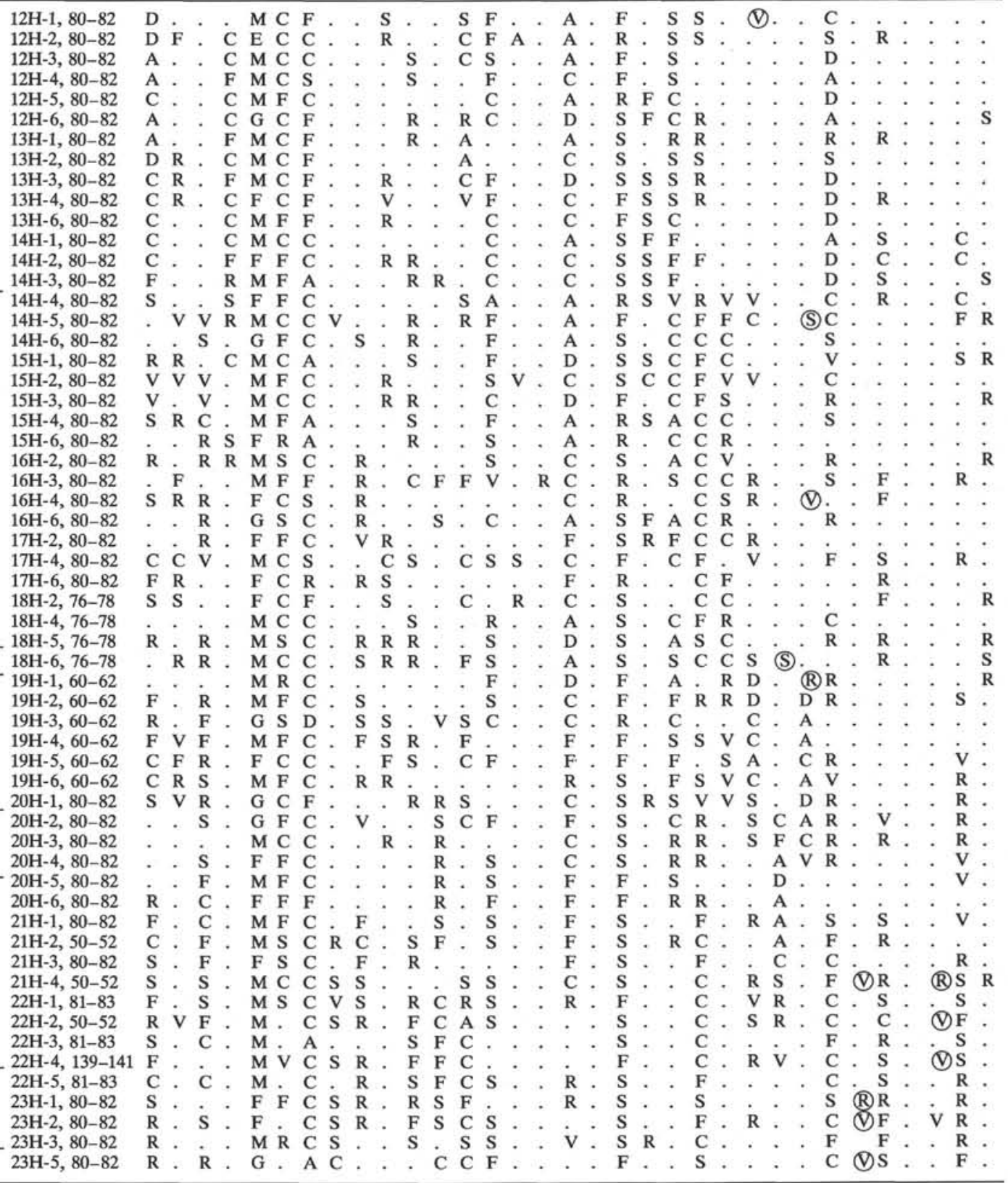

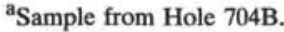


Table 3 (continued).

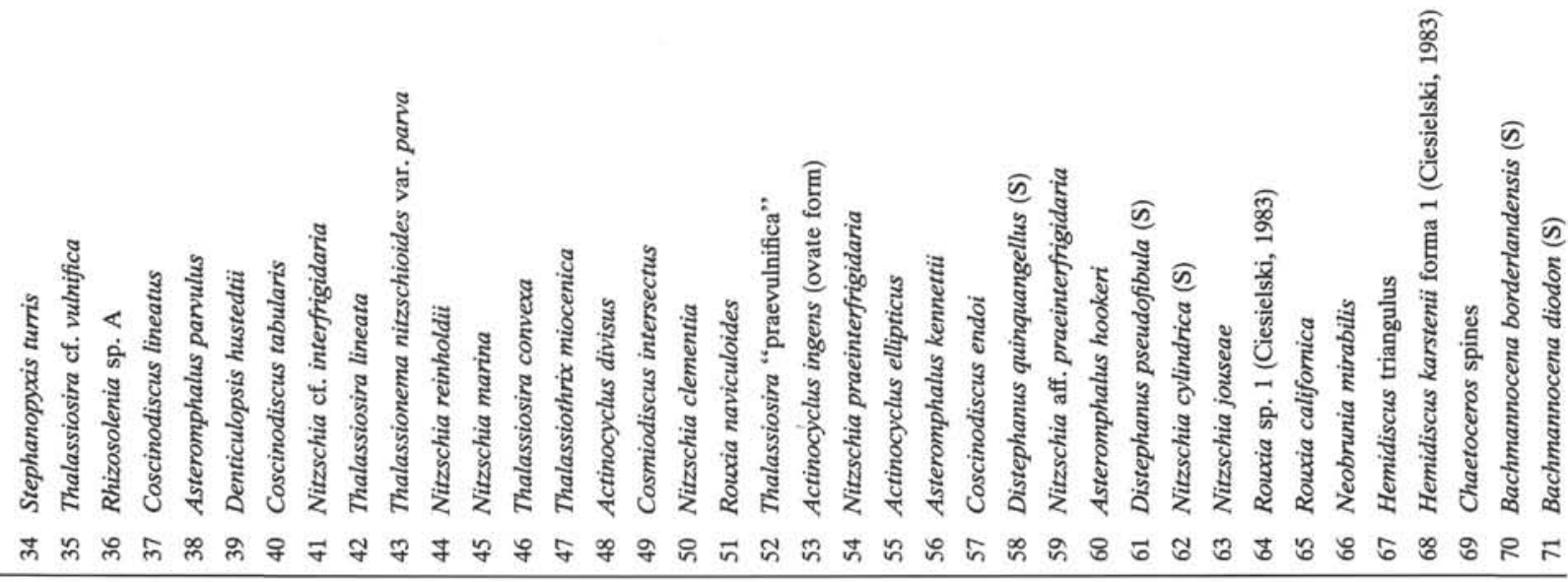

$1 \mathrm{H}-2,78-80$

$1 \mathrm{H}-3,78-80$

$1 \mathrm{H}-5,78-80$

2H-1, 40-42

2H-2, 130-134

2H-3, 130-134

2H-4, 130-134

2H-5, 130-134

$2 \mathrm{H}-7,40-42$

$3 \mathrm{H}-1,80-84$

$3 \mathrm{H}-2,80-84$

$3 \mathrm{H}-3,26-30$

$3 \mathrm{H}-4,26-30$

$3 \mathrm{H}-5,26-30$

$3 \mathrm{H}-6,26-30$

B4H-1, 120-122

B4H-2, 120-122

B4H-3, 120-122

B4H-4, 120-122

B4H-5, 120-122

$5 \mathrm{H}-1,50-52$

$5 \mathrm{H}-2,50-52$

$5 \mathrm{H}-3,50-52$

$5 \mathrm{H}-4,50-52$

$5 \mathrm{H}-5,80-82$

$6 \mathrm{H}-1,84-86$

$6 \mathrm{H}-2,84-86$

$6 \mathrm{H}-3,84-86$

$6 \mathrm{H}-4,84-86$

$6 \mathrm{H}-5,84-86$

$6 \mathrm{H}-6,84-86$

(1) $\dot{\mathrm{S}}$

7H-1, 59-61

$7 \mathrm{H}-2,59-61$

7H-3, 59-61

7H-4, 59-61

$7 \mathrm{H}-5,59-61$

$7 \mathrm{H}-6,59-61$

$8 \mathrm{H}-1,80-82$

$8 \mathrm{H}-2,80-82$

$8 \mathrm{H}-3,80-82$

$8 \mathrm{H}-4,80-82$

$8 \mathrm{H}-5,80-82$

$8 \mathrm{H}-6,80-82$

$9 \mathrm{H}-1,80-82$

$9 \mathrm{H}-2,80-82$

9H-3, 80-82

$9 \mathrm{H}-4,80-82$

9H-5, 80-82

9H-6, 80-82

$10 \mathrm{H}-1,79-81$

$10 \mathrm{H}-2,79-81$

$10 \mathrm{H}-3,79-81$

$10 \mathrm{H}-4,79-81$

10H-5, 79-81

$10 \mathrm{H}-6,79-8$

$11 \mathrm{H}-1,80-82$

$11 \mathrm{H}-2,80-82$

$11 \mathrm{H}-3,80-82$

$11 \mathrm{H}-4,80-82$ 

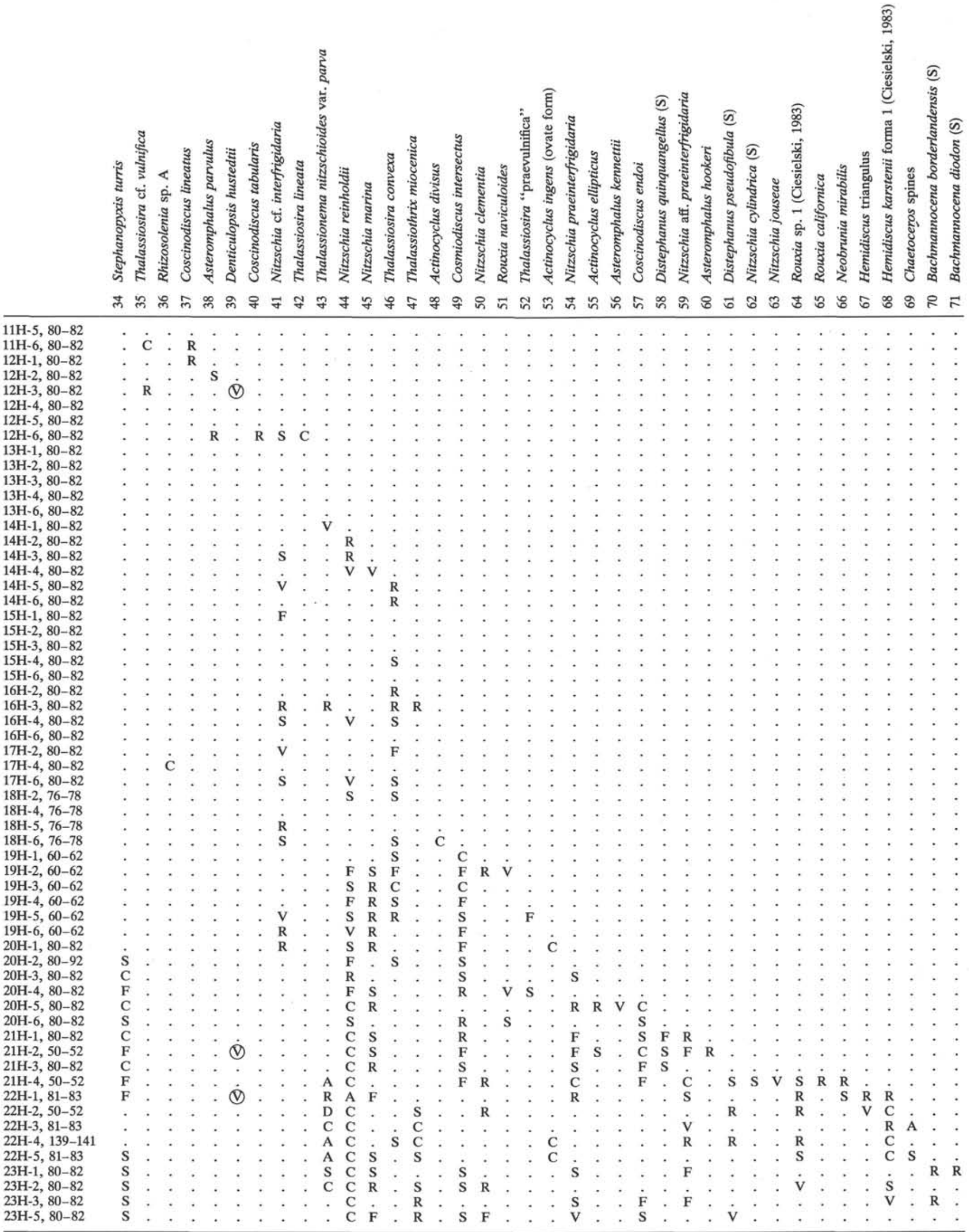
Species location index

Index number is the column in which species appears.

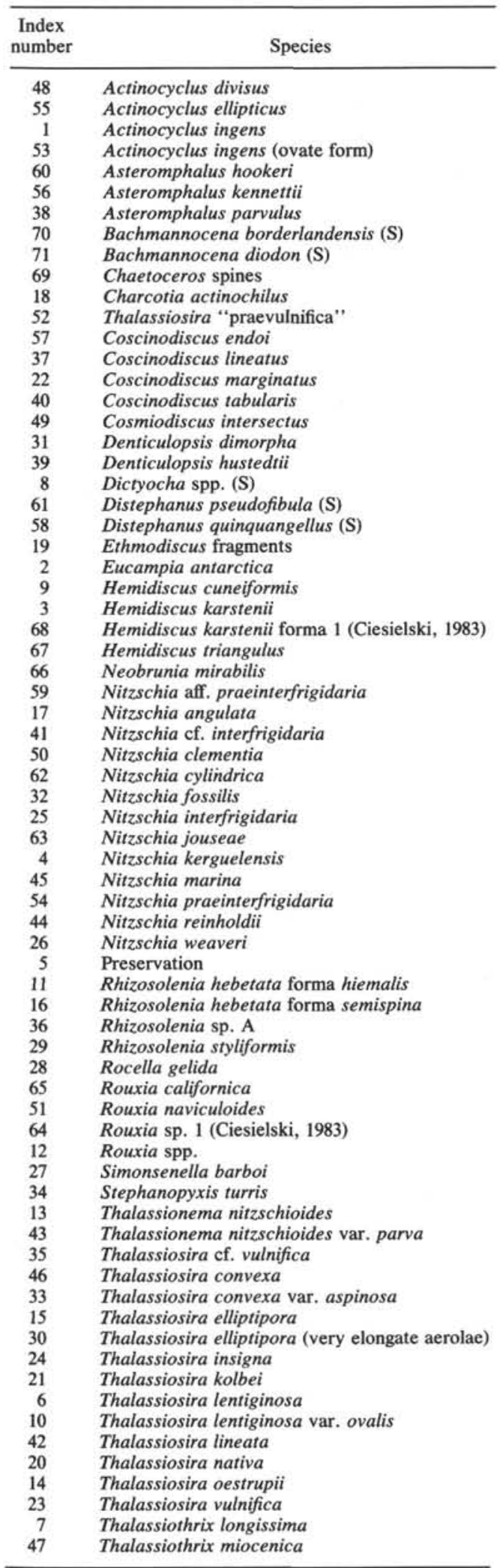

Note: $\mathrm{V}=$ very rare $\mathrm{R}=\operatorname{rare} ; \mathrm{S}=$ sparse $; \mathrm{F}=$ frequent $\mathrm{C}$ = common $; \mathrm{A}=$ abundant $\mathrm{D}=$ dominant $; \mathrm{P}=$ poor; $\mathrm{VP}$ = very poor $\mathbf{P}=$ poor $; \mathrm{F}=$ fair $; \mathrm{M}=$ moderate $; \mathbf{G}=$ good; $\mathrm{E}=$ excellent; ? = questionably present; . = not present. 


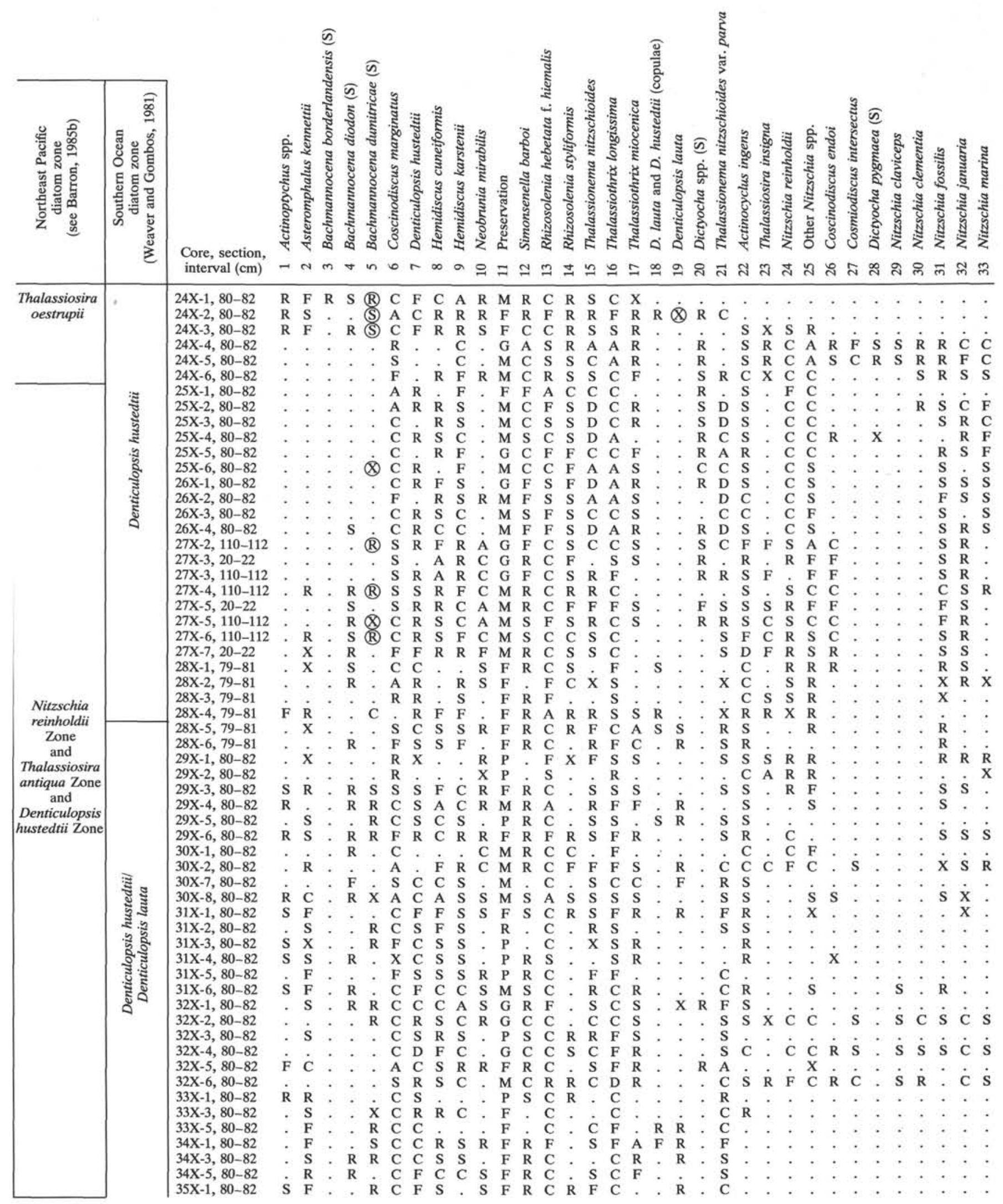




\begin{tabular}{|c|c|c|c|c|c|c|c|c|c|c|c|c|c|c|c|c|c|c|c|c|c|c|c|c|c|c|c|c|c|c|}
\hline 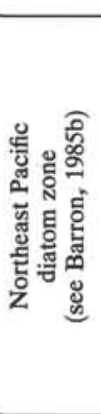 & 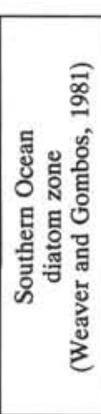 & $\begin{array}{l}\text { Core, section, } \\
\text { interval }(\mathrm{cm})\end{array}$ & & 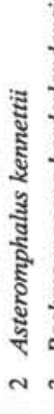 & 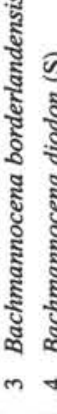 & 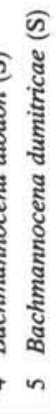 & 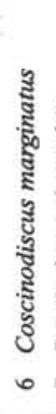 & 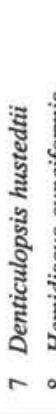 & & 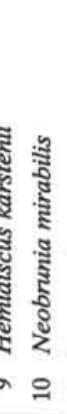 & 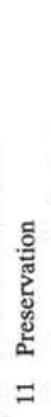 & 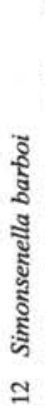 & 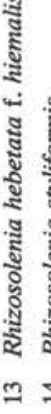 & 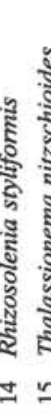 & 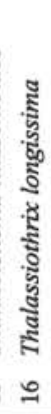 & 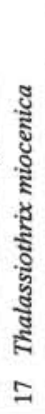 & 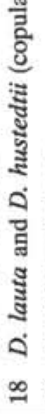 & 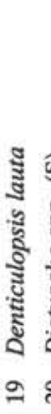 & 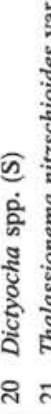 & 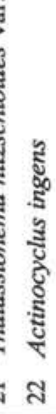 & 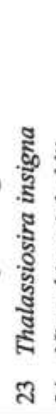 & 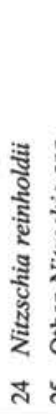 & 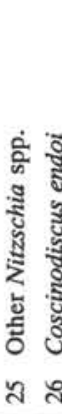 & & & & 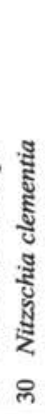 & 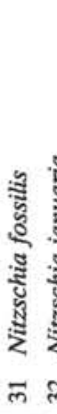 & & \\
\hline & 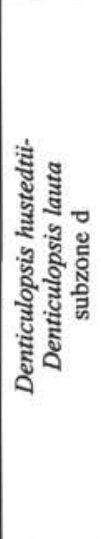 & $\begin{array}{l}35 X-3,80-82 \\
35 X-5,80-82 \\
36 X-1,80-82 \\
37 X-1,80-82 \\
37 X-3,80-82 \\
39 X-2,80-82 \\
39 X-5,80-82 \\
40 X-2,80-82 \\
41 X-2,80-82 \\
41 X-5,80-82 \\
42 X-2,80-82 \\
42 X-6,80-82 \\
43 X-3,79-81 \\
43 X-5,79-81 \\
44 X-1,80-82 \\
45 X-2,81-83 \\
46 X-2,80-82 \\
46 X-3,80-82 \\
46 X-4,80-82\end{array}$ & 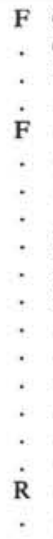 & $\begin{array}{l}\mathrm{C} \\
\mathrm{C} \\
\mathrm{S} \\
: \\
: \\
\vdots \\
\dot{\mathrm{R}} \\
\mathrm{S} \\
\mathrm{S} \\
: \\
: \\
: \\
: \\
:\end{array}$ & 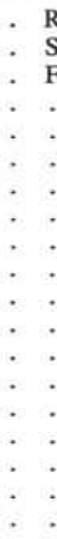 & $\begin{array}{l}\mathrm{S} \\
\mathrm{R} \\
\mathrm{C} \\
\mathrm{F} \\
\mathrm{R} \\
\dot{ } \\
\dot{ } \\
\dot{\mathrm{R}} \\
\dot{\mathrm{R}} \\
\dot{\mathrm{S}} \\
\dot{ } \\
\end{array}$ & $\begin{array}{l}\text { C } \\
\text { C } \\
\text { F } \\
\text { C } \\
\text { C } \\
\text { C } \\
\text { S } \\
\text { F } \\
\text { S } \\
\text { S } \\
\text { S } \\
\text { S } \\
\text { C } \\
\text { S } \\
\text { S } \\
\text { C } \\
\text { S } \\
\text { S } \\
\text { S }\end{array}$ & $\begin{array}{ll}\text { C } & \\
\text { F } & \\
\text { C } & \text { I } \\
\text { C } & 1 \\
\text { F } & \text { I } \\
\text { C } & \text { I } \\
\text { C } & \\
\text { C } \\
\text { C } \\
\text { A } \\
\text { A } \\
\text { C } \\
\text { C } \\
\text { F } \\
\text { C } \\
\text { C } \\
\text { S } \\
\text { R } \\
\text { C }\end{array}$ & 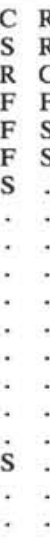 & 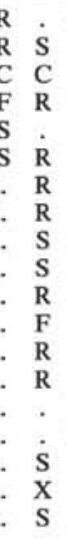 & $\begin{array}{l}\text { M } \\
F \\
F \\
M \\
F \\
F \\
F \\
F \\
M \\
M \\
M \\
F \\
F \\
P \\
F \\
M \\
P \\
F \\
M\end{array}$ & $\begin{array}{l}\text { F } \\
C \\
C \\
\text { F } \\
\text { R } \\
\text { S } \\
\text { S } \\
\text { F } \\
\text { S } \\
\text { F } \\
\text { S } \\
\text { S } \\
\text { R } \\
\text { S } \\
\text { S } \\
\text { C } \\
\text { R } \\
\text {. }\end{array}$ & $\begin{array}{l}\text { C } \\
\text { C } \\
\text { C } \\
\text { C } \\
\text { C } \\
\text { C } \\
\text { F } \\
\text { C } \\
\text { C } \\
\text { C } \\
\text { F } \\
\text { F } \\
\text { S } \\
\text { S } \\
\text { F } \\
\text { S } \\
\text { R } \\
\text { R } \\
\text { S }\end{array}$ & 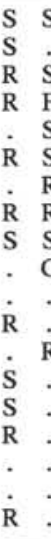 & $\begin{array}{l}\text { C } \\
\text { C } \\
\text { C } \\
\text { C } \\
\text { F } \\
\text { A } \\
\text { F } \\
\text { F } \\
\text { F } \\
\text { C } \\
\text { S } \\
\text { F } \\
\text { S } \\
\text { S } \\
\text { F } \\
\text { F } \\
\text { S } \\
\text { S } \\
\text { S }\end{array}$ & $\begin{array}{l}\text { S } \\
\dot{R} \\
\text { F } \\
\text { C } \\
\text { S } \\
\dot{R} \\
\therefore \\
\therefore \\
: \\
: \\
: \\
: \\
:\end{array}$ & $\begin{array}{l}\cdot \\
\dot{C} \\
\text { S } \\
\dot{S} \\
\text { S } \\
\text { R } \\
\text { R } \\
\text { S } \\
\text { S } \\
\text { S } \\
\text { F } \\
\text { F } \\
\text { S } \\
\text { S } \\
\text { R } \\
\text { R } \\
\text { S }\end{array}$ & $\begin{array}{l}X \\
X \\
S \\
\cdot \\
\dot{R} \\
R \\
\text { S } \\
R \\
R \\
R \\
R \\
C \\
F \\
\text { S } \\
F \\
\text { S } \\
R \\
C\end{array}$ & $\begin{array}{l} \\
\dot{\mathrm{S}} \\
\mathrm{R} \\
\dot{\mathrm{R}} \\
\dot{\mathrm{R}} \\
\dot{\mathrm{R}} \\
\dot{\mathrm{R}} \\
\dot{ } \\
\dot{ } \\
\dot{5}\end{array}$ & $\begin{array}{l}\text { R } \\
\text { R } \\
\text { L } \\
\text { A } \\
\text { R } \\
\text { S } \\
\text { C } \\
\text { C } \\
\text { A } \\
\text { A } \\
\text { A } \\
\text { C } \\
\text { C } \\
\text { F } \\
\text { A } \\
\text { C } \\
\text { S } \\
\text { S } \\
\text { F }\end{array}$ & $\begin{array}{l}: \\
: \\
: \\
: \\
: \\
: \\
: \\
: \\
: \\
:\end{array}$ & $\begin{array}{l}\cdot \\
: \\
: \\
: \\
: \\
: \\
: \\
: \\
: \\
: \\
: \\
:\end{array}$ & 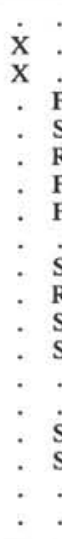 & & $\begin{array}{l}\dot{5} \\
\vdots \\
\vdots \\
\vdots\end{array}$ & $\begin{array}{l}: \\
: \\
: \\
: \\
:\end{array}$ & $\begin{array}{l}: \\
\vdots \\
\vdots \\
\vdots\end{array}$ & $\begin{array}{l}\mathrm{R} \\
: \\
\vdots \\
\vdots \\
\vdots \\
\vdots \\
\vdots \\
\vdots \\
: \\
: \\
: \\
:\end{array}$ & i & : \\
\hline
\end{tabular}


Table 4-Part B.

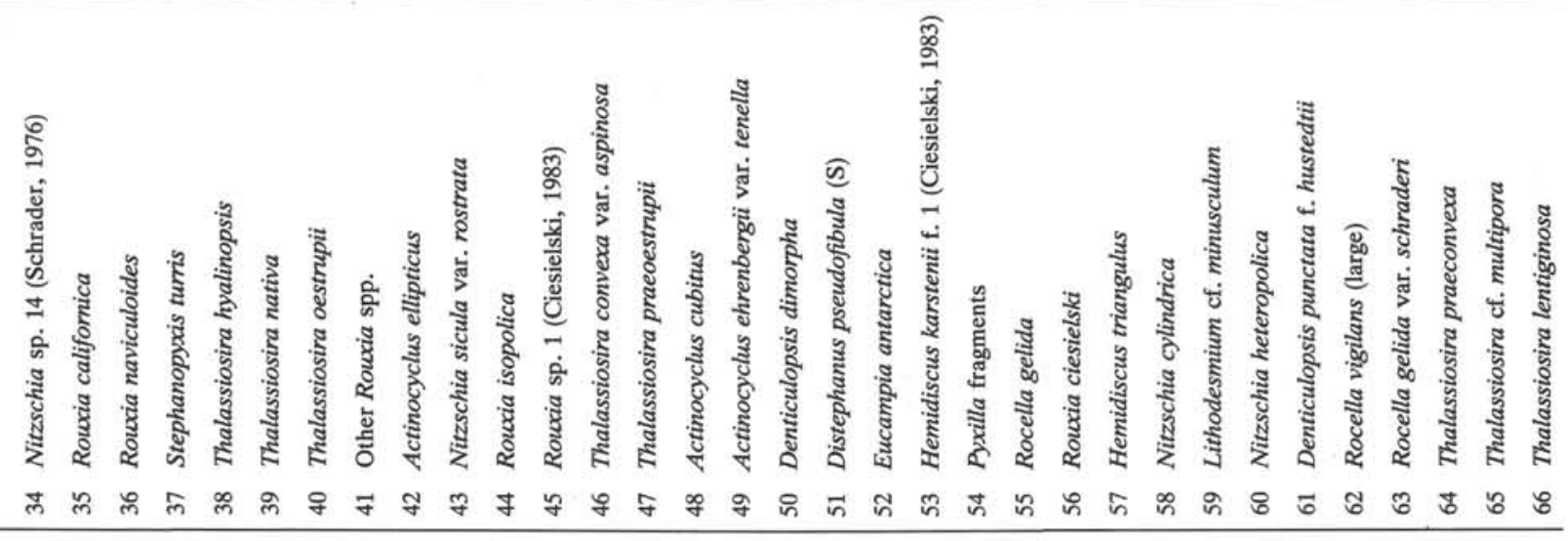

24X-1, 80-82

24X-2, 80-82

24X-3, 80-82

$24 \mathrm{X}-4,80-82$

24X-5, 80-82

$24 \mathrm{X}-6,80-82$

$25 \mathrm{X}-1,80-82$

25X-2, 80-82

$25 \mathrm{X}-3,80-82$

25X-4, 80-82

$25 \mathrm{X}-5,80-82$

25X-6, 80-82

26X-1, 80-82

26X-2, 80-82

$26 \mathrm{X}-3,80-82$

26X-4, 80-82

27X-2, 110-112

27X-3, 20-22

$27 \mathrm{X}-3,110-112$

$27 \mathrm{X}-5,20-22$

27X-5, 110-112

$27 \mathrm{X}-6,110-112$

27X-7, 20-22

28X-1, 79-81

28X-2, 79-81

$28 \mathrm{X}-3,79-81$

$28 \mathrm{X}-4,79-81$

28X-5, 79-81

28X-6, 79-81

29X-1, 80-82

29X-2, 80-82

29X-3, 80-82

$29 \mathrm{X}-4,80-82$

29X-5, 80-82

29X-6, 80-82

$30 \mathrm{X}-1,80-82$

\begin{tabular}{llllllllllllll}
$\dot{\mathrm{C}}$ & $\dot{\mathrm{F}}$ & $\dot{\mathrm{R}}$ & $\dot{\mathrm{R}}$ & $\dot{\mathrm{R}}$ & $\dot{\mathrm{C}}$ & $\dot{\mathrm{C}}$ & $\dot{\mathrm{R}}$ \\
\hline
\end{tabular}

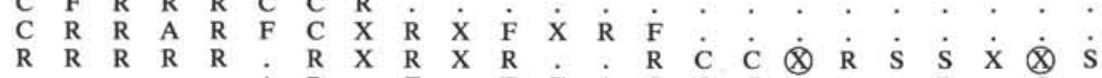

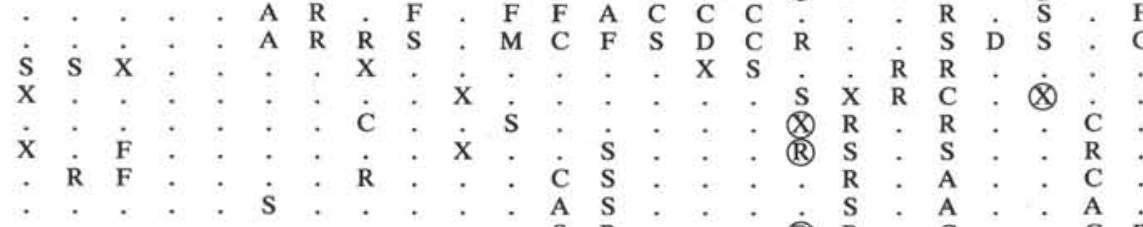

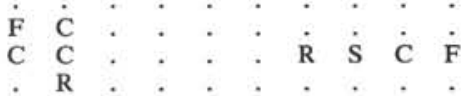

$30 \mathrm{X}-2,80-82$

$30 \mathrm{X}-7,80-82$

$30 \mathrm{X}-8,80-82$

$31 \mathrm{X}-1,80-82$

$31 \mathrm{X}-2,80-82$

$31 \mathrm{X}-3,80-82$

$31 \mathrm{X}-4,80-82$

$31 \mathrm{X}-5,80-82$

$31 \mathrm{X}-6,80-82$

$32 \mathrm{X}-1,80-82$

$32 \mathrm{X}-2,80-82$

$32 \mathrm{X}-3,80-82$

$32 \mathrm{X}-4,80-82$

$32 \mathrm{X}-5,80-82$

$32 \mathrm{X}-6,80-82$

$33 \mathrm{X}-1,80-82$

$33 \mathrm{X}-3,80-82$

$33 \mathrm{X}-5,80-82$

$34 \mathrm{X}-1,80-82$

$34 \mathrm{X}-3,80-82$

$34 \mathrm{X}-5,80-82$

$35 \mathrm{X}-1,80-82$

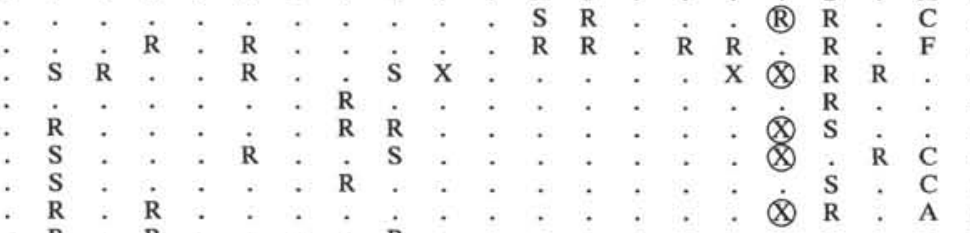

$\dot{\otimes}$

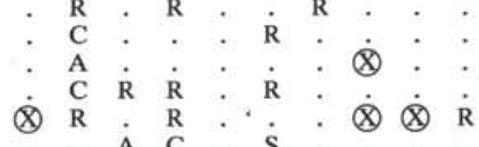

$\therefore \quad{ }_{C}^{\mathrm{A}}{ }_{F}^{\mathrm{C}}: \dot{\mathrm{S}}^{\mathrm{R}}: \dot{\mathrm{A}}^{\otimes} \mathrm{R}^{\mathrm{R}} \dot{\mathrm{S}}$

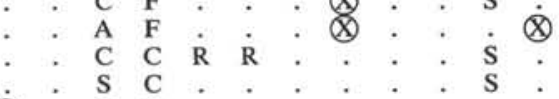

$\otimes$

$\begin{array}{ll}\mathrm{S} & \mathrm{C} \\ \mathrm{C} & \mathrm{C} \\ \mathrm{F} & \mathrm{R}\end{array}$

$\dot{\otimes} \dot{x} \quad \dot{x}$

$\dot{\otimes}$

(B) ${ }_{\mathrm{X}}^{\mathrm{R}} \cdot \dot{\otimes}$

( $)$.

(৫)

()

$\otimes$

(B)

()

$\dot{\otimes}$ 


\begin{tabular}{|c|c|c|c|c|c|c|c|c|c|c|c|c|c|c|c|c|c|c|c|c|c|c|c|c|c|c|c|c|c|c|c|c|c|}
\hline & 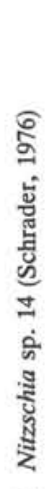 & 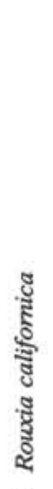 & 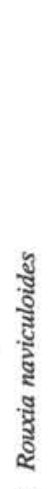 & 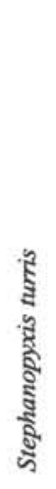 & 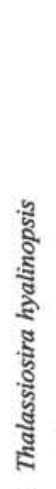 & 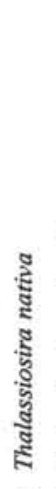 & 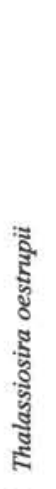 & 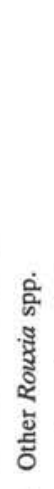 & 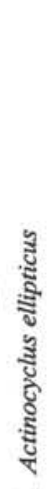 & 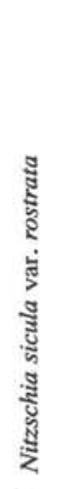 & 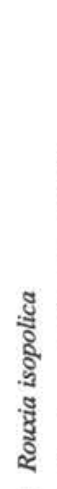 & 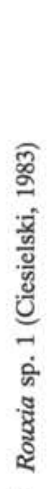 & 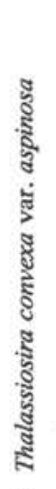 & 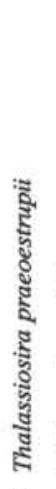 & 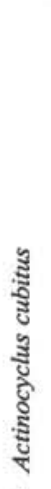 & 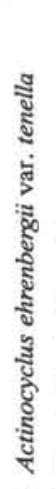 & 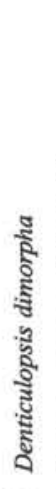 & 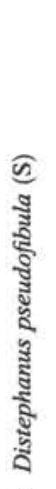 & 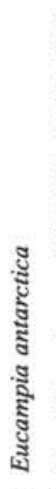 & 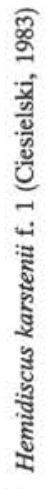 & 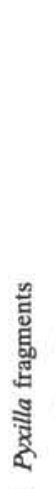 & 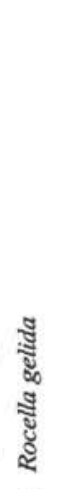 & 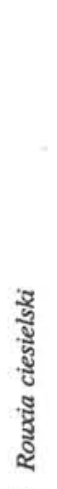 & 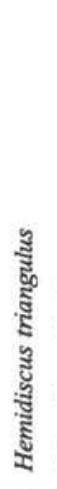 & 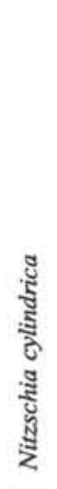 & 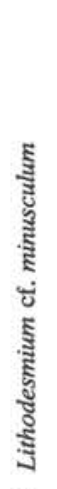 & 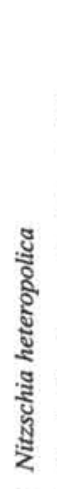 & 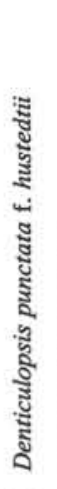 & 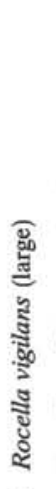 & 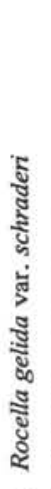 & 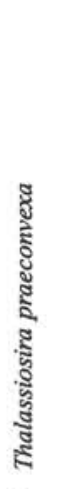 & 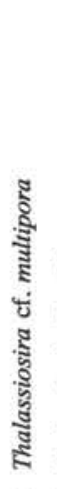 & 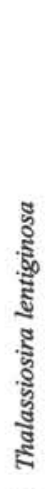 \\
\hline & স & 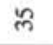 & లి & n & $\stackrel{\infty}{\infty}$ & ले & f & $F$ & F & $\mathscr{q}$ & J & y & $f$ & f & $\stackrel{\infty}{\mathfrak{d}}$ & $g$ & ๓ి & $\bar{n}$ & กี & $\tilde{n}$ & ஜี & $\approx$ & ஜ̊ & n & $\infty_{n}$ & ถి & 8 & 5 & ช్ర & 8 & पे & 8 & 8 \\
\hline $35 \mathrm{X}-3,80-82$ & . & . & . & . & . & $\mathbf{S}$ & . & . & $\mathbf{R}$ & . & . & . & . & . & . & . & $\mathrm{R}$ & . & . & . & . & . & • & . & . & & . & . & . & . & . & . & \\
\hline $35 \mathrm{X}-5,80-82$ & . & . & . & . & . & . & . & . & . & . & . & . & . & . & . & . & & . & . & . & . & . & . & . & . & & . & . & . & . & . & . & . \\
\hline $36 \mathrm{X}-1,80-82$ & . & . & & . & . & . & . & . & $\mathbf{R}$ & . & . & . & . & . & . & . & $\mathrm{x}$ & . & . & . & . & . & . & . & . & . & . & 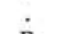 & . & . & . & . & \\
\hline $37 \mathrm{X}-1,80-82$ & . & . & . & . & . & . & . & . & . & . & . & . & . & . & . & . & $\mathbf{R}$ & . & . & . & . & . & . & . & . & . & . & $\mathrm{R}$ & . & . & . & . & . \\
\hline $37 \mathrm{X}-3,80-82$ & . & . & & . & . & . & . & . & $\mathbf{R}$ & . & . & . & . & . & . & . & $\mathrm{X}$ & . & . & . & . & . & . & . & . & . & . & . & . & . & . & . & \\
\hline $39 \times-2,80-82$ & . & . & . & . & . & . & . & . & . & . & . & . & . & . & . & . & $\mathrm{x}$ & . & . & . & . & . & . & . & . & . & . & . & . & . & . & . & . \\
\hline $39 X-5,80-82$ & . & . & . & . & . & . & . & . & . & . & . & . & . & . & . & . & & . & . & . & . & . & . & . & . & . & . & . & . & . & . & . & \\
\hline $40 \mathrm{X}-2,80-82$ & . & . & . & . & . & . & . & . & . & . & . & . & . & . & . & . & S & 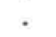 & . & . & $\mathrm{x}$ & . & . & . & . & & . & . & . & . & . & . & . \\
\hline$X-2,80-82$ & . & . & . & . & . & . & . & . & . & . & . & . & . & . & . & . & $\mathbf{R}$ & . & & . & . & . & 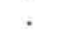 & . & . & . & . & . & . & . & . & . & \\
\hline $41 X-5,80-82$ & . & . & . & . & . & . & . & . & . & . & . & . & . & . & . & . & $\mathbf{R}$ & . & $\mathbf{R}$ & . & . & . & . & . & . & & . & . & . & . & . & . & . \\
\hline$X-2,80-82$ & . & . & . & . & . & . & . & . & & . & . & . & . & . & . & . & $\mathbf{R}$ & . & $\mathrm{R}$ & . & . & . & . & . & . & . & . & . & . & . & . & . & \\
\hline $42 \mathrm{X}-6,80-82$ & . & . & . & . & . & . & . & . & $\mathrm{R}$ & . & . & . & . & . & . & . & $\mathbf{R}$ & . & . & . & . & . & . & . & . & . & . & . & . & . & . & . & . \\
\hline $43 \times-3,79-81$ & . & . & . & . & . & . & . & . & . & . & . & . & . & . & . & . & D & . & . & . & . & . & . & . & . & . & . & . & . & . & . & . & \\
\hline $43 X-5,79-81$ & . & . & . & . & . & . & . & . & . & . & . & . & . & . & . & . & D & . & . & . & . & . & . & . & . & . & . & . & . & . & . & . & . \\
\hline $44 \mathrm{X}-1,80-82$ & . & . & . & . & . & . & . & . & . & . & . & . & . & . & . & . & $\mathrm{s}$ & . & . & . & . & . & . & . & . & . & . & . & . & . & . & . & \\
\hline $45 X-2,81-83$ & . & . & . & . & . & . & . & . & . & . & . & . & . & . & . & . & D & . & . & . & . & & . & . & . & . & . & . & . & . & . & . & . \\
\hline $46 \mathrm{X}-2,80-82$ & . & . & . & . & . & . & . & . & . & . & . & . & . & . & . & . & D & . & . & . & . & $\mathrm{X}$ & 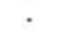 & . & . & . & & . & & & & & \\
\hline $46 \mathrm{X}-3,80-82$ & . & . & . & . & . & . & . & . & . & . & . & . & . & $\cdot$ & . & . & D & . & . & . & . & $\mathrm{S}$ & . & . & . & . & . & . & $\otimes$ & $\otimes$ & . & . & . \\
\hline $46 \mathrm{X}-4,80-82$ & . & . & . & 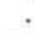 & . & . & & . & $\cdot$ & . & . & & & & . & . & D & . & . & . & . & $\mathrm{X}$ & . & . & . & . & . & . & . & . & . & . & \\
\hline
\end{tabular}




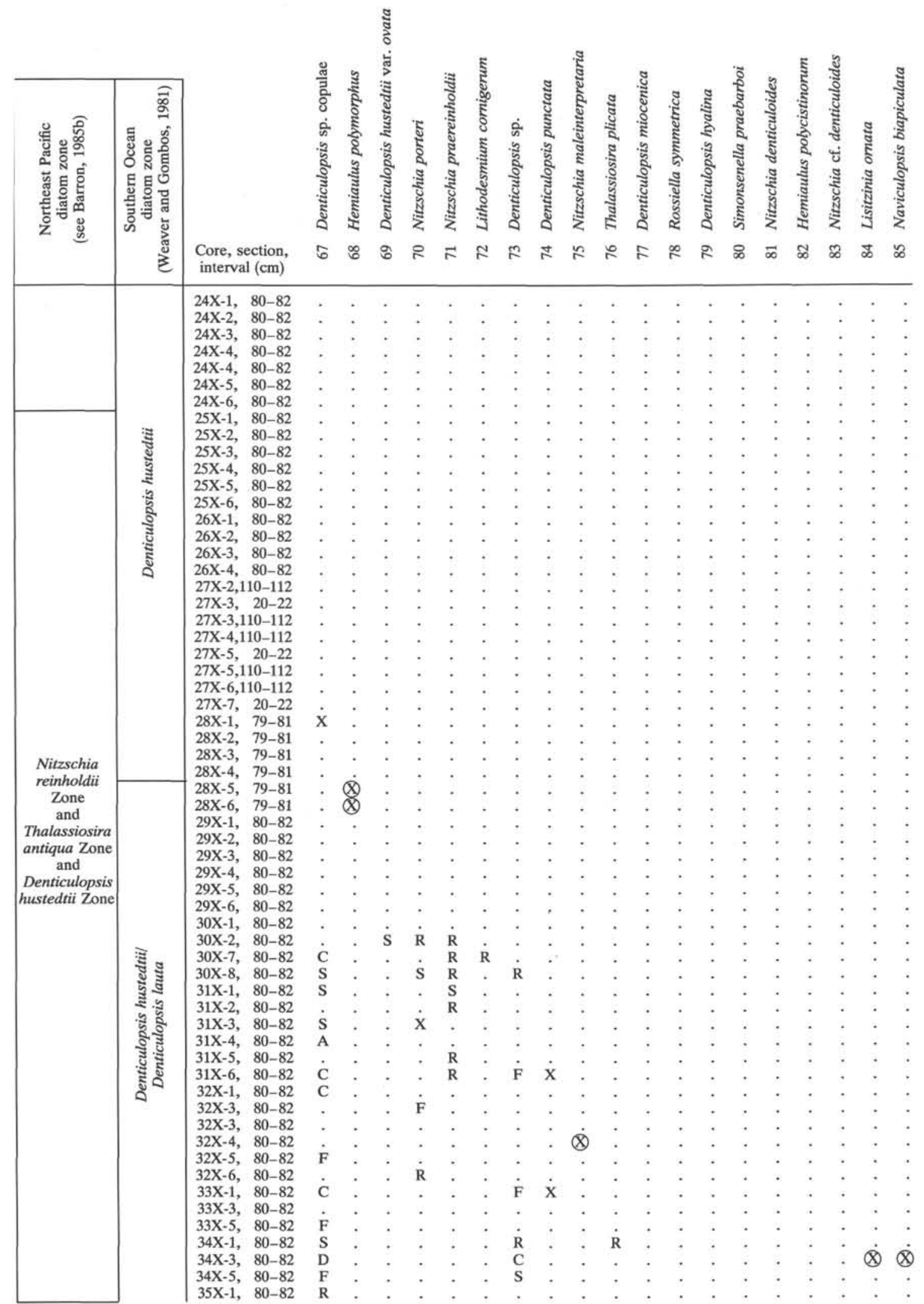


Table 4-Part C.

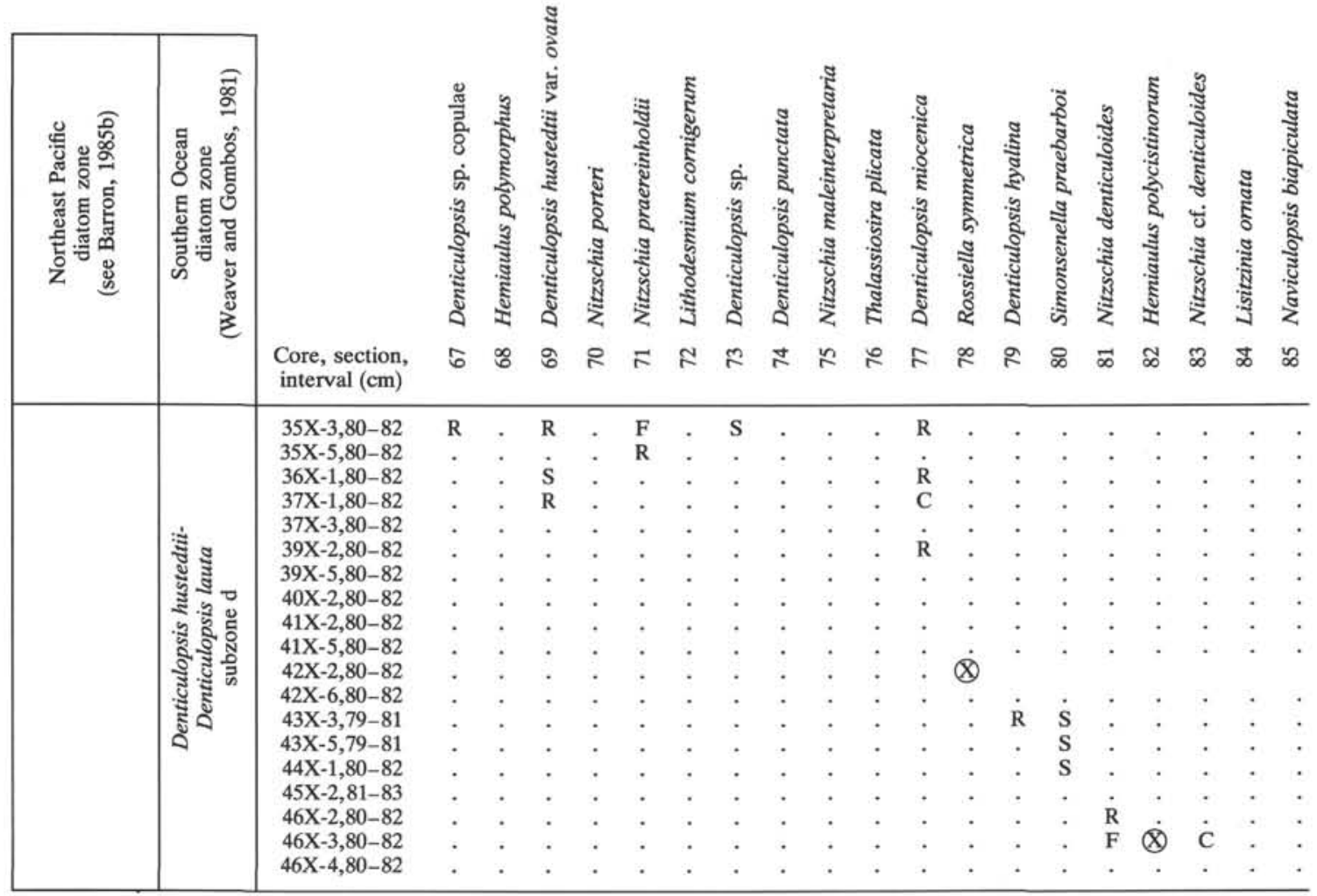


Species location index

Index number is the column in which species appears.

\begin{tabular}{|c|c|}
\hline $\begin{array}{l}\text { Index } \\
\text { number }\end{array}$ & Species \\
\hline 48 & Actinocyclus cubitus \\
\hline 49 & Actinocyclus ehrenbergii var. tenella \\
\hline 42 & Actinocyclus ellipticus \\
\hline 22 & Actinocyclus ingens \\
\hline 1 & Actinoptychus spp. \\
\hline 2 & Asteromphalus kennettii \\
\hline 3 & Bachmannocena borderlandensis (S) \\
\hline 4 & Bachmannocena diodon $(\mathrm{S})$ \\
\hline 5 & Bachmannocena dumitricae (S) \\
\hline 26 & Coscinodiscus endoi \\
\hline 6 & Coscinodiscus marginatus \\
\hline 27 & Cosmiodiscus intersectus \\
\hline 50 & Denticulopsis dimorpha \\
\hline 7 & Denticulopsis hustedtii \\
\hline 69 & Denticulopsis hustedtii var. ovata \\
\hline 79 & Denticulopsis hyalina \\
\hline 19 & Denticulopsis lauta \\
\hline 18 & $D$. lauta and D. hustedtii (copulae) \\
\hline 77 & Denticulopsis miocenica \\
\hline 74 & Denticulopsis punctata \\
\hline 61 & Denticulopsis punctata f. hustedtii \\
\hline 73 & Denticulopsis sp. \\
\hline 67 & Denticulopsis sp. copulae \\
\hline 28 & Dictyocha pygmaea (S) \\
\hline 20 & Dictyocha spp. (S) \\
\hline 51 & Distephanus pseudofibula (S) \\
\hline 52 & Eucampia antarctica \\
\hline 82 & Hemiaulus polycistinorum \\
\hline 68 & Hemiaulus polymorphus \\
\hline 8 & Hemidiscus cuneiformis \\
\hline 9 & Hemidiscus karstenii \\
\hline 53 & Hemidiscus karstenii f. 1 (Ciesielski, 1983) \\
\hline 57 & Hemidiscus triangulus \\
\hline 84 & Lisitzinia ornata \\
\hline 59 & Lithodesmium cf. minusculum \\
\hline 72 & Lithodesmium cornigerum \\
\hline 85 & Naviculopsis biapiculata \\
\hline 10 & Neobrunia mirabilis \\
\hline 83 & Nitzschia cf. denticuloides \\
\hline 29 & Nitzschia claviceps \\
\hline 30 & Nitzschia clementia \\
\hline 58 & Nitzschia cylindrica \\
\hline 81 & Nitzschia denticuloides \\
\hline 31 & Nitzschia fossilis \\
\hline 60 & Nitzschia heteropolica \\
\hline 32 & Nitzschia januaria \\
\hline 75 & Nitzschia maleinterpretaria \\
\hline 33 & Nitzschia marina \\
\hline 70 & Nitzschia porteri \\
\hline 71 & Nitzschia praereinholdii \\
\hline 24 & Nitzschia reinholdii \\
\hline 43 & Nitzschia sicula var. rostrata \\
\hline 34 & Nitzschia sp. 14 (Schrader, 1976) \\
\hline 25 & Other Nitzschia spp. \\
\hline 41 & Other Rouxia spp. \\
\hline 11 & Preservation \\
\hline 54 & Pyxilla fragments \\
\hline 13 & Rhizosolenia hebetata f. hiemalis \\
\hline 14 & Rhizosolenia styliformis \\
\hline 55 & Rocella gelida \\
\hline 63 & Rocella gelida var. schraderi \\
\hline 62 & Rocella vigilans (large) \\
\hline 78 & Rossiella symmetrica \\
\hline 35 & Rouxia californica \\
\hline 56 & Rouxia ciesielski \\
\hline 44 & Rouxia isopolica \\
\hline 36 & Rouxia naviculoides \\
\hline 45 & Rouxia sp. 1 (Ciesielski, 1983) \\
\hline 12 & Simonsenella barboi \\
\hline 80 & Simonsenella praebarboi \\
\hline 37 & Stephanopyxis turris \\
\hline 15 & Thalassionema nitzschioides \\
\hline 21 & Thalassionema nitzschioides var. parva \\
\hline 65 & Thalassiosira cf. multipora \\
\hline
\end{tabular}

Species location index

Index number is the column in which species appears.

\begin{tabular}{cl}
$\begin{array}{c}\text { Index } \\
\text { number }\end{array}$ & \multicolumn{1}{c}{ Species } \\
\hline 46 & Thalassiosira convexa var. aspinosa \\
38 & Thalassiosira hyalinopsis \\
23 & Thalassiosira insigna \\
66 & Thalassiosira lentiginosa \\
39 & Thalassiosira nativa \\
40 & Thalassiosira oestrupii \\
76 & Thalassiosira plicata \\
64 & Thalassiosira praeconvexa \\
47 & Thalassiosira praeoestrupii \\
16 & Thalassiothrix longissima \\
17 & Thalassiothrix miocenica \\
\hline
\end{tabular}

Note: $\mathrm{X}=$ very rare; $\mathrm{R}=$ rare; $\mathrm{S}=$ sparse; $\mathrm{F}=$ frequent; $\mathrm{C}=$ common; $\mathrm{A}=$ abundant; $\mathrm{D}=$ dominant; $\mathrm{P}=$ poor; $\mathrm{F}=$ fair; $\mathrm{M}=$ moderate $\mathrm{G}=$ good $; \mathrm{E}=$ excellent; ? = questionably present; . = not present. 


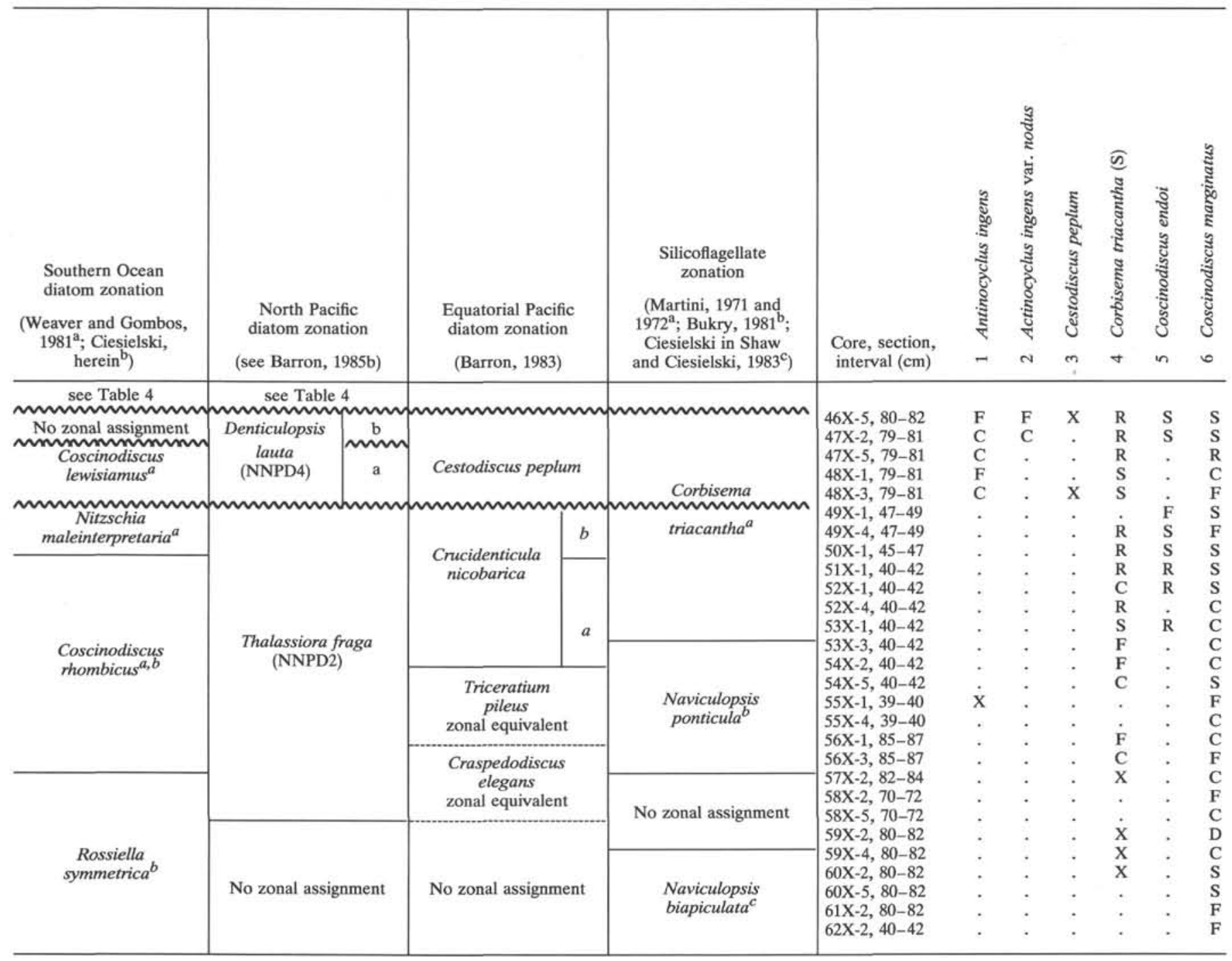


Table 4-Part E.

Core, section, interval $(\mathrm{cm})$

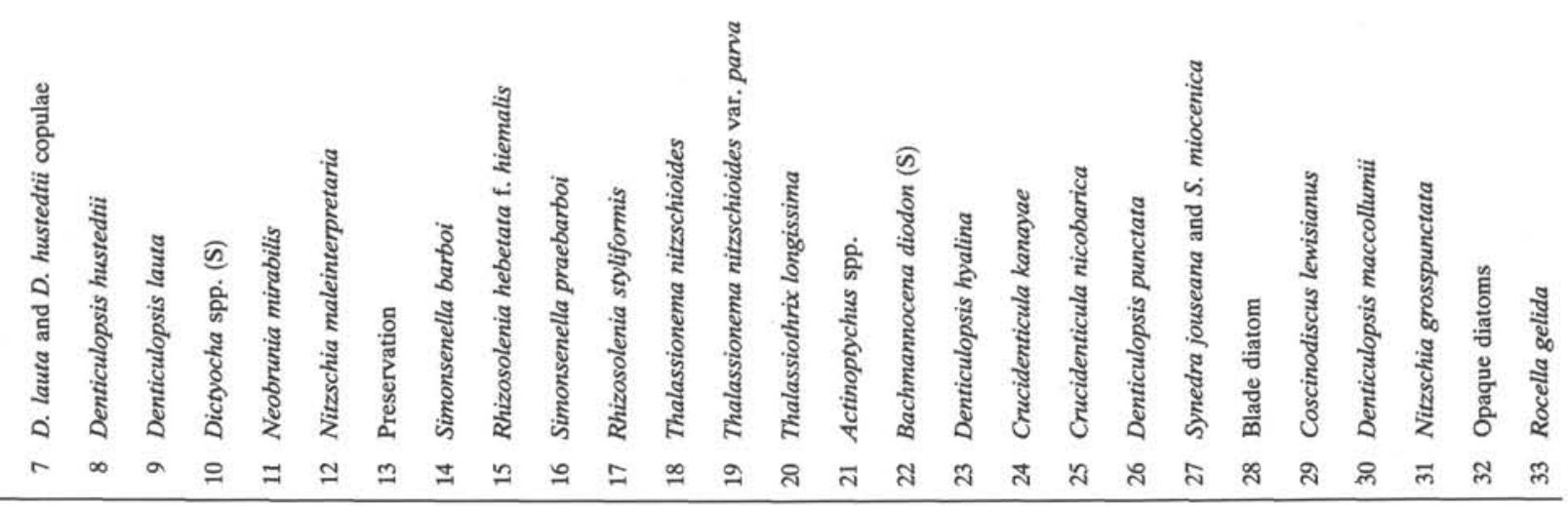

\begin{tabular}{|c|c|c|c|c|c|c|c|c|c|c|c|c|c|c|c|c|c|c|c|c|c|c|c|c|c|c|}
\hline $46 X-5,80-82$ & R & F & $\mathbf{S}$ & F & $\mathbf{R}$ & $\mathrm{X}$ & F & F & $\mathbf{S}$ & F & $\mathbf{R}$ & $\mathrm{S}$ & S & $\mathbf{S}$ & . & & 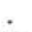 & & & . & - & 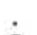 & . & . & . & . \\
\hline $47 X-2,79-81$ & . & $\mathbf{S}$ & S & $\mathrm{S}$ & F & . & M & F & $\mathbf{S}$ & S & . & C & A & F & $\mathbf{R}$ & $\mathbf{S}$ & $\mathrm{X}$ & $\mathrm{X}$ & C & $\mathrm{X}$ & $\mathbf{R}$ & S & . & . & $\dot{0}$ & $\dot{x}$ \\
\hline $47 X-5,79-81$ & . & . & $\mathbf{R}$ & $\mathbf{F}$ & . & . & F & $\mathrm{S}$ & $\mathbf{R}$ & F & $\mathbf{R}$ & $\mathbf{R}$ & $\mathbf{R}$ & $\mathbf{F}$ & $\mathbf{R}$ & . & . & - & F & . & $\mathbf{R}$ & C & $\mathbf{R}$ & $\mathrm{S}$ & $\mathbf{R}$ & R \\
\hline $48 X-1,79-81$ & . & . & S & $\mathbf{R}$ & . & . & M & C & F & S & $\mathbf{S}$ & S & $\mathbf{S}$ & C & $\mathbf{F}$ & . & . & . & $\mathbf{S}$ & . & $\mathbf{R}$ & C & $\mathbf{R}$ & $\mathrm{S}$ & $\mathrm{X}$ & S \\
\hline $48 X-3,79-81$ & . & $\mathrm{X}$ & $\mathbf{R}$ & S & . & R & M & . & . & $\mathrm{F}$ & . & F & $\mathbf{S}$ & $\mathbf{S}$ & $\mathrm{S}$ & . & . & . & $\mathbf{S}$ & . & S & $\mathrm{F}$ & $\mathrm{S}$ & C & $\mathbf{R}$ & . \\
\hline $49 X-1,47-49$ & . & . & . & $\dot{0}$ & $\dot{-}$ & . & $\mathbf{P}$ & F & S & $\mathbf{R}$ & . & $\mathbf{R}$ & $\mathbf{R}$ & $\mathbf{R}$ & $\mathbf{R}$ & . & . & . & . & . & $\mathbf{F}$ & $\mathbf{S}$ & $\mathbf{R}$ & F & $\mathbf{R}$ & . \\
\hline $49 X-4,47-49$ & . & . & . & $\mathbf{S}$ & $\mathbf{R}$ & $\mathbf{R}$ & G & . & $\mathbf{R}$ & . & . & . & C & $\mathbf{R}$ & $\mathrm{R}$ & . & . & C & D & . & $\mathrm{S}$ & C & $\mathbf{R}$ & $\mathbf{R}$ & $\mathbf{R}$ & . \\
\hline $50 \times-1,45-47$ & . & - & . & $\mathbf{R}$ & . & F & $\mathrm{P}$ & . & . & . & . & . & . & S & $\mathrm{S}$ & . & . & $\mathrm{R}$ & $\mathbf{R}$ & . & $\mathrm{S}$ & $\mathrm{S}$ & . & . & $\mathbf{R}$ & . \\
\hline $51 X-1,40-42$ & & . & . & $\mathbf{S}$ & $\mathbf{R}$ & $S$ & F & . & $\mathbf{R}$ & . & . & $\mathrm{R}$ & $\mathrm{F}$ & S & F & . & . & $S$ & $\mathrm{~F}$ & . & $\mathrm{R}$ & $\mathbf{S}$ & $\mathbf{R}$ & . & . & . \\
\hline $52 X-1,40-42$ & (S) & . & . & C & $\mathbf{R}$ & R & F & . & - & . & . & $\mathrm{S}$ & C & F & . & . & . & $\mathrm{R}$ & C & . & $\mathbf{S}$ & $\mathbf{S}$ & $\mathrm{X}$ & . & . & . \\
\hline $52 X-4,40-42$ & . & - & . & $\mathrm{S}$ & . & . & $\mathbf{P}$ & . & . & $\mathbf{R}$ & , & $\mathbf{S}$ & $\mathrm{S}$ & $\mathbf{S}$ & $\mathrm{S}$ & . & . & . & . & . & $\mathbf{R}$ & S & . & . & . & S \\
\hline $53 X-1,40-42$ & . & . & . & $\mathrm{F}$ & $\mathrm{F}$ & . & G & . & R & $\mathrm{S}$ & . & S & . & S & C & . & . & . & . & . & $\mathbf{R}$ & $S$ & . & . & . & $\mathbf{R}$ \\
\hline $53 X-3,40-42$ & . & . & . & $\mathbf{S}$ & $\mathrm{R}$ & $\mathrm{X}$ & M & . & $\mathrm{X}$ & $\mathrm{S}$ & . & $\mathrm{S}$ & S & $\mathrm{F}$ & C & . & . & . & $\dot{8}$ & . & $\mathrm{R}$ & $\mathrm{S}$ & $\mathbf{R}$ & . & . & . \\
\hline $54 X-2,40-42$ & . & . & . & . & F & F & E & . & . & F & . & S & $\mathrm{X}$ & $\mathrm{S}$ & F & . & . & . & $\mathrm{C}$ & S & $\mathrm{R}$ & F & . & . & . & . \\
\hline $54 X-5,40-42$ & . & . & . & . & . & $\mathbf{R}$ & M & . & . & F & . & . & . & $\mathbf{S}$ & . & . & . & . & . & . & $\mathrm{S}$ & S & . & . & - & $\dot{8}$ \\
\hline $55 X-1,39-40$ & . & . & . & . & . & . & $\mathbf{P}$ & . & . & $\mathbf{R}$ & . & . & . & . & . & . & . & . & . & . & $\mathbf{F}$ & $\mathrm{S}$ & . & - & . & $\mathrm{S}$ \\
\hline $55 X-4,39-40$ & . & . & . & . & . & . & F & . & $\mathrm{X}$ & $\mathbf{R}$ & . & $\mathrm{S}$ & $\mathrm{F}$ & $\mathbf{R}$ & $\mathbf{R}$ & . & . & . & . & . & C & $\mathrm{R}$ & . & . & . & $\mathbf{R}$ \\
\hline $56 X-1,85-87$ & . & . & . & . & . & $\mathbf{R}$ & G & $\mathbf{R}$ & $X$ & F & . & . & . & S & . & . & . & . & . & . & A & C & . & . & . & $\mathbf{S}$ \\
\hline $56 X-3,85-87$ & . & . & . & $\mathbf{R}$ & . & . & F & $\mathrm{S}$ & $\mathbf{R}$ & $\mathbf{S}$ & . & $\mathrm{S}$ & . & $\mathrm{R}$ & . & . & . & . & . & . & C & $\mathrm{S}$ & . & . & . & $\mathbf{R}$ \\
\hline $57 X-2,82-84$ & . & . & . & . & . & . & M & . & . & $\mathbf{R}$ & . & . & . & $\mathbf{R}$ & $\mathrm{R}$ & . & . & . & . & . & C & $\mathrm{S}$ & . & . & . & C \\
\hline $58 X-2,70-72$ & . & . & . & . & . & . & M & $\mathbf{R}$ & $\mathbf{R}$ & $\mathrm{X}$ & . & $\mathbf{R}$ & $\mathrm{X}$ & $\mathrm{R}$ & . & . & . & . & . & . & C & $\mathbf{S}$ & . & . & . & . \\
\hline $58 X-5,70-72$ & . & . & . & . & . & . & M & . & . & . & - & F & S & $\mathrm{F}$ & R & . & . & . & . & . & C & $\mathrm{S}$ & . & . & . & . \\
\hline $59 X-2,80-82$ & . & , & . & . & . & . & M & . & $\mathbf{R}$ & $\mathbf{R}$ & . & $\mathrm{S}$ & . & F & $\mathbf{R}$ & . & . & . & . & . & C & C & . & . & . & . \\
\hline $59 X-4,80-82$ & . & . & . & . & . & . & M & . & . & $\mathbf{S}$ & . & $\mathbf{R}$ & $\mathrm{S}$ & $\mathbf{R}$ & . & . & . & . & - & . & C & $\mathrm{S}$ & . & . & . & . \\
\hline $60 X-2,80-82$ & . & . & . & . & . & . & G & . & $\mathbf{R}$ & $\mathrm{S}$ & . & $\mathrm{S}$ & $\mathbf{R}$ & . & $\mathbf{R}$ & . & . & . & . & . & C & $\mathrm{S}$ & . & . & - & - \\
\hline $60 X-5,80-82$ & . & . & . & . & . & . & $\mathrm{F}$ & . & $\mathbf{R}$ & - & . & $\mathrm{X}$ & $\mathrm{S}$ & . & $\mathbf{F}$ & . & . & . & - & . & C & $\mathbf{R}$ & . & . & - & . \\
\hline $61 X-2,80-82$ & . & . & . & . & . & . & F & . & . & $\mathbf{R}$ & . & $\mathbf{F}$ & $\mathbf{R}$ & $\mathrm{S}$ & . & . & . & . & . & . & C & F & . & . & . & $\dot{0}$ \\
\hline $62 X-2,40-42$ & . & . & . & . & . & . & $\mathrm{P}$ & . & . & $\mathrm{S}$ & . & $\mathbf{F}$ & . & . & . & . & . & . & . & . & S & $\mathbf{R}$ & . & . & . & S \\
\hline
\end{tabular}




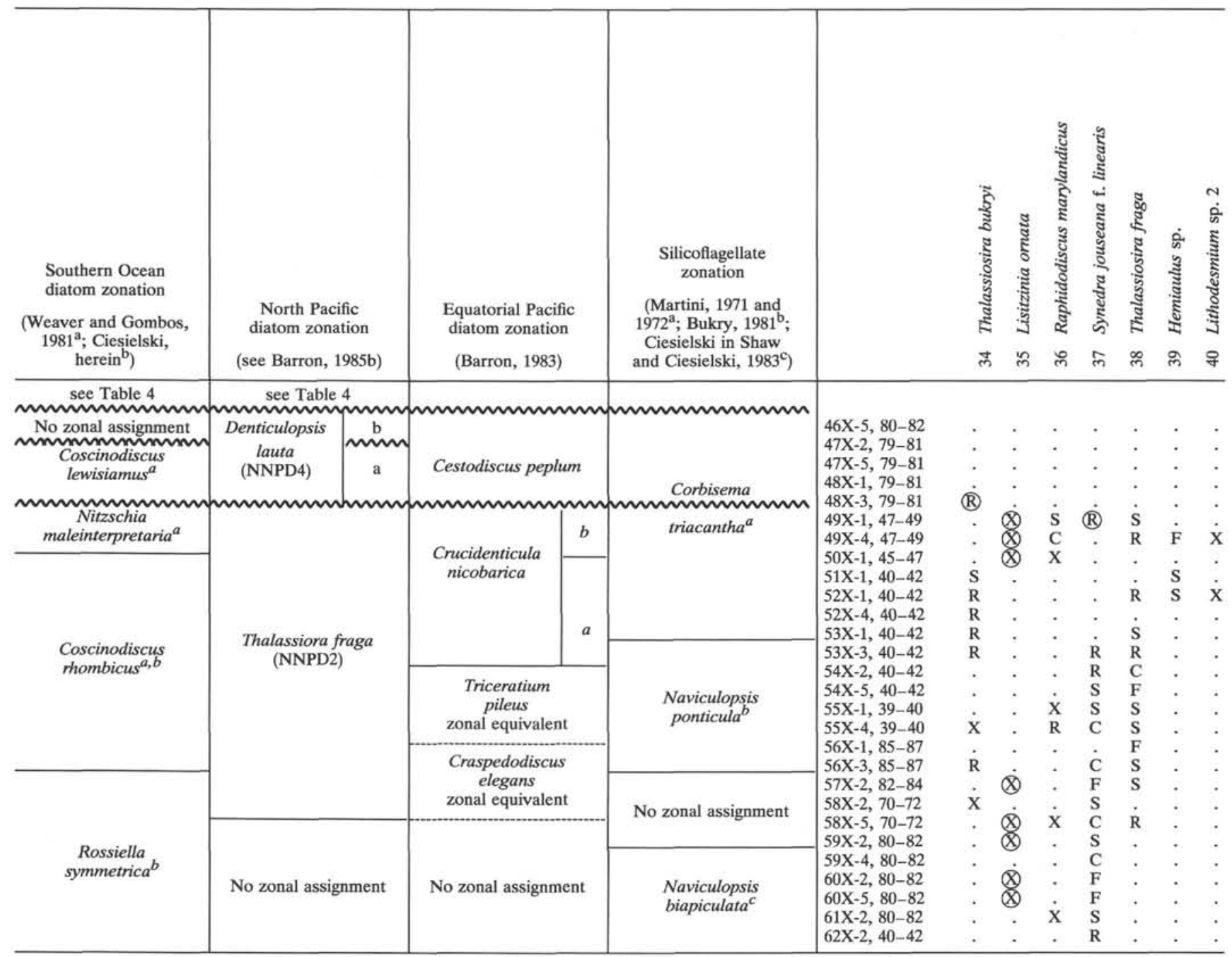




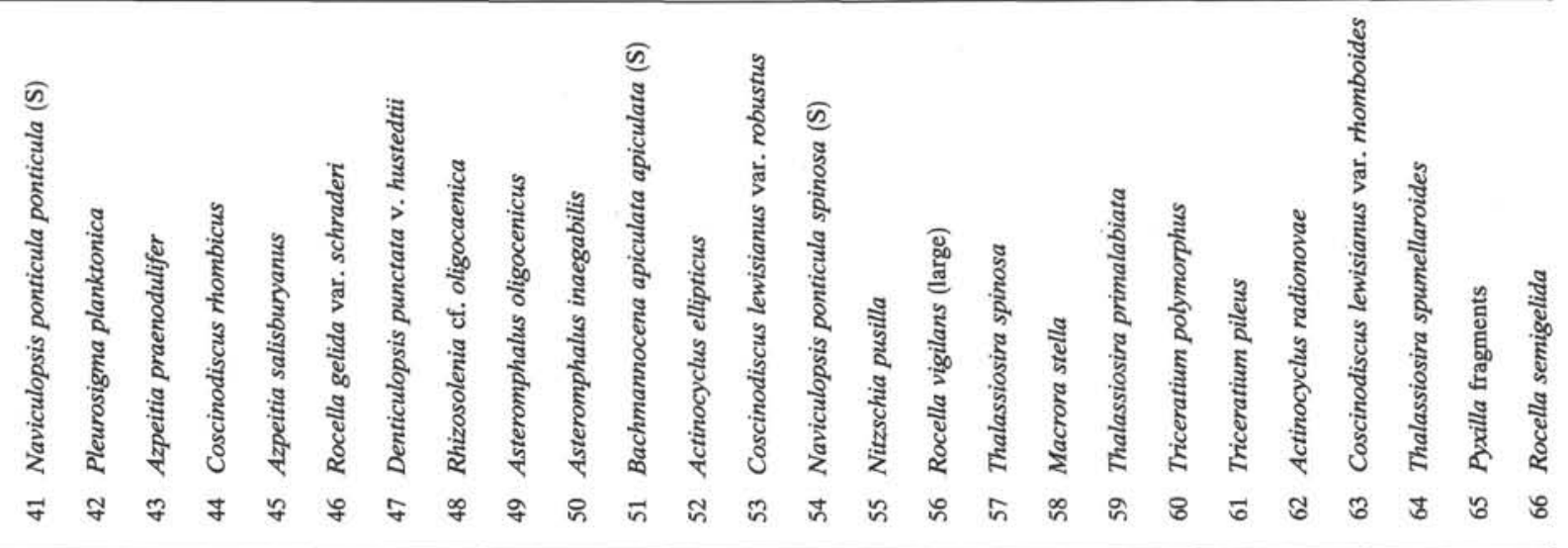

$46 \mathrm{X}-5,80-82$

$47 \mathrm{X}-2,79-81$

$47 X-5,79-81$

$48 \mathrm{X}-1,79-81$

$48 \mathrm{X}-3,79-8$

$49 \mathrm{X}-1,47-49$

$49 \mathrm{X}-4,47-49$

50X-1, 45-47

51X-1, 40-42

$52 \mathrm{X}-1,40-42$

$52 X-4,40-42$

$53 \mathrm{X}-1,40-42$

$53 \mathrm{X}-3,40-42$

$54 \mathrm{X}-2,40-42$

$54 \mathrm{X}-5,40-42$

$55 \mathrm{X}-1,39-40$

$55 \mathrm{X}-4,39-40$

$56 \mathrm{X}-1,85-87$

$56 \mathrm{X}-3,85-87$

57X-2, 82-84

58X-2, 70-72

$58 \mathrm{X}-5,70-72$

$59 X-2,80-82$

$59 \mathrm{X}-4,80-82$

60X-2, 80-82

$60 \mathrm{X}-5,80-82$

$61 \mathrm{X}-2,80-82$

$62 X-2,40-42$ 
Table 4-Part G.

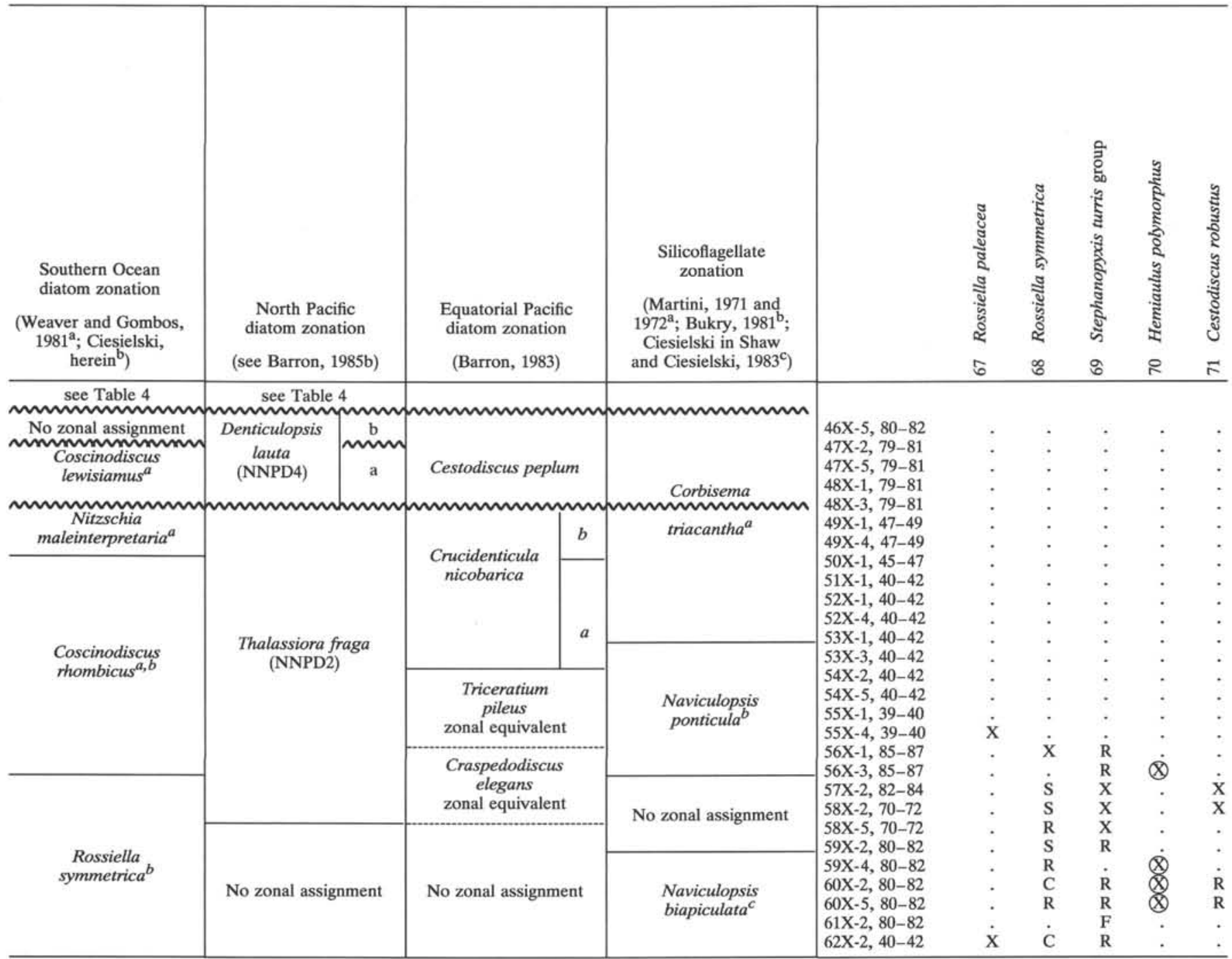


Table 4-Part G.

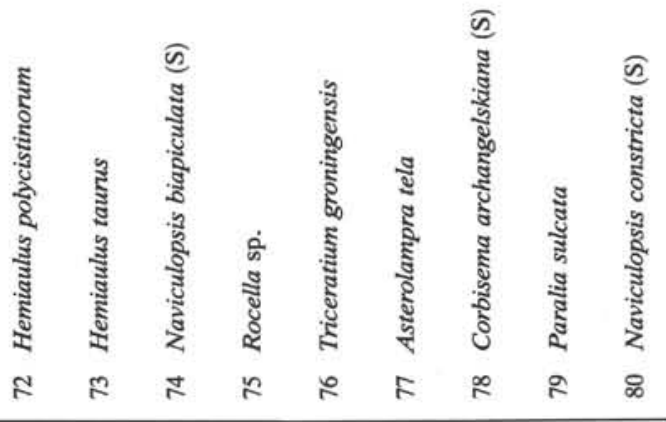

\begin{tabular}{|c|c|c|c|c|c|c|c|c|}
\hline 58 & & & & & & & & \\
\hline$(-2,79-81$ & . & . & . & . & . & . & . & . \\
\hline $47 \mathrm{X}-5,79-81$ & . & . & . & . & . & . & . & . \\
\hline $48 \mathrm{X}-1,79-81$ & . & . & . & . & . & . & . & . \\
\hline $48 \mathrm{X}-3,79-81$ & . & . & . & . & . & . & . & . \\
\hline $49 \times-1,47-49$ & . & . & . & . & . & . & . & . \\
\hline $49 \times-4,47-49$ & . & . & . & . & . & . & . & - \\
\hline $50 \times-1,45-47$ & . & . & . & . & . & . & . & . \\
\hline $51 X-1,40-42$ & . & . & . & . & . & . & . & . \\
\hline $52 X-1,40-42$ & . & . & . & . & . & . & . & . \\
\hline $52 X-4,40-42$ & . & . & . & . & . & . & . & . \\
\hline $53 \times-1,40-42$ & . & . & . & . & . & . & . & . \\
\hline $53 X-3,40-42$ & . & . & . & . & . & . & . & . \\
\hline $54 X-2,40-42$ & . & . & . & . & . & . & . & . \\
\hline $54 X-5,40-42$ & . & . & . & . & . & . & . & . \\
\hline $55 \mathrm{X}-1,39-40$ & . & . & . & . & . & . & . & . \\
\hline $55 X-4,39-40$ & . & . & . & . & . & . & . & \\
\hline $56 \mathrm{X}-1,85-87$ & . & . & . & . & . & . & . & . \\
\hline $56 \mathrm{X}-3,85-87$ & & & & & & & . & \\
\hline $57 \mathrm{X}-2,82-84$ & $\otimes$ & $\otimes$ & $\otimes$ & $\mathrm{x}$ & $\otimes$ & . & . & . \\
\hline $58 X-2,70-72$ & . & $\cdot$ & & . & . & & & \\
\hline $\begin{array}{l}58 X-5,70-72 \\
59 X-2,80-82\end{array}$ & : & : & $\begin{array}{l}\mathrm{X} \\
\mathrm{X}\end{array}$ & . & : & S & ? & . \\
\hline $59 \mathrm{X}-4,80-82$ & & & s & $\mathrm{x}$ & & . & & \\
\hline $60 X-2,80-82$ & ß & (B) & C & . & (S) & ${ }^{\circ}$ & (1) & $\mathrm{x}$ \\
\hline $60 X-5,80-$ & & & C & . & & $\mathrm{x}$ & $\otimes$ & . \\
\hline $61 X-2,80-82$ & . & . & $\mathrm{S}$ & & $\mathbf{R}$ & . & . & . \\
\hline $62 X-2,40-42$ & . & . & $\mathrm{s}$ & $\mathrm{x}$ & $\mathbf{R}$ & . & . & . \\
\hline
\end{tabular}


Species location index

Index number is the column in which species appears.

\begin{tabular}{|c|c|}
\hline $\begin{array}{l}\text { Index } \\
\text { number }\end{array}$ & Species \\
\hline 52 & Actinocyclus ellipticus \\
\hline 1 & Actinocyclus ingens \\
\hline 2 & Actinocyclus ingens var. nodus \\
\hline 62 & Actinocyclus radionovae \\
\hline 21 & Actinoptychus spp. \\
\hline 77 & Asterolampra tela \\
\hline 50 & Asteromphalus inaegabilis \\
\hline 49 & Asteromphalus oligocenicus \\
\hline 43 & Azpeitia praenodulifer \\
\hline 45 & Azpeitia salisburyanus \\
\hline 51 & Bachmannocena apiculata apiculata (S) \\
\hline 22 & Bachmannocena diodon (S) \\
\hline 28 & Blade diatom \\
\hline 3 & Cestodiscus peplum \\
\hline 71 & Cestodiscus robustus \\
\hline 78 & Corbisema archangelskiana (S) \\
\hline 4 & Corbisema triacantha $(\mathrm{S})$ \\
\hline 5 & Coscinodiscus endoi \\
\hline 29 & Coscinodiscus lewisianus \\
\hline 63 & Coscinodiscus lewisianus var. rhomboides \\
\hline 53 & Coscinodiscus lewisianus var, robustus \\
\hline 6 & Coscinodiscus marginatus \\
\hline 44 & Coscinodiscus rhombicus \\
\hline 24 & Crucidenticula kanayae \\
\hline 25 & Crucidenticula nicobarica \\
\hline 8 & Denticulopsis hustedtii \\
\hline 23 & Denticulopsis hyalina \\
\hline 9 & Denticulopsis lauta \\
\hline 7 & D. lauta and $D$. hustedtii copulae \\
\hline 30 & Denticulopsis maccollumii \\
\hline 26 & Denticulopsis punctata \\
\hline 47 & Denticulopsis punctata v. hustedtii \\
\hline 10 & Dictyocha spp. (S) \\
\hline 72 & Hemiaulus polycistinorum \\
\hline 70 & Hemiaulus polymorphus \\
\hline 39 & Hemiaulus sp. \\
\hline 73 & Hemiaulus taurus \\
\hline 35 & Lisitzinia ornata \\
\hline 40 & Lithodesmium sp. 2 \\
\hline 58 & Macrora stella \\
\hline 74 & Naviculopsis biapiculata (S) \\
\hline 80 & Naviculopsis constricta (S) \\
\hline 41 & Naviculopsis ponticula ponticula (S) \\
\hline 54 & Naviculopsis ponticula spinosa (S) \\
\hline 11 & Neobrunia mirabilis \\
\hline 31 & Nitzschia grossepunctata \\
\hline 12 & Nitzschia maleinterpretaria \\
\hline 55 & Nitzschia pusilla \\
\hline 32 & Opaque diatoms \\
\hline 79 & Paralia sulcata \\
\hline 42 & Pleurosigma planktonica \\
\hline 13 & Preservation \\
\hline 65 & Pyxilla fragments \\
\hline 36 & Raphidodiscus marylandicus \\
\hline 15 & Rhizosolenia hebetata f. hiemalis \\
\hline 48 & Rhizosolenia cf. oligocaenica \\
\hline 17 & Rhizosolenia styliformis \\
\hline 33 & Rocella gelida \\
\hline 46 & Rocella gelida var. schraderi \\
\hline 66 & Rocella semigelida \\
\hline 75 & Rocella sp. \\
\hline 56 & Rocella vigilans (large) \\
\hline 67 & Rossiella paleacea \\
\hline 68 & Rossiella symmetrica \\
\hline 14 & Simonsenella barboi \\
\hline 16 & Simonsenella praebarboi \\
\hline 69 & Stephanopyxis turris group \\
\hline 27 & Synedra jouseana and $S$. miocenica \\
\hline 37 & Synedra jouseana $\mathrm{f}$. linearis \\
\hline 18 & Thalassionema nitzschioides \\
\hline 19 & Thalassionema nitzschioides var. parva \\
\hline 34 & Thalassiosira bukryi \\
\hline 38 & Thalassiosira fraga \\
\hline 59 & Thalassiosira primalabiata \\
\hline
\end{tabular}

\section{Species location index}

Index number is the column in which species appears.

\begin{tabular}{cl}
\hline $\begin{array}{c}\text { Index } \\
\text { number }\end{array}$ & \multicolumn{1}{c}{ Species } \\
\hline 57 & Thalassiosira spinosa \\
64 & Thalassiosira spumellaroides \\
20 & Thalassiothrix longissima \\
76 & Triceratium groningensis \\
61 & Triceratium pileus \\
60 & Triceratium polymorphus \\
\hline
\end{tabular}

Note: $\mathrm{X}=$ very rare; $\mathrm{R}=$ rare; $\mathrm{S}=$ sparse $\mathrm{F}=$ frequent; $\mathrm{C}=$ common; $\mathrm{A}=$ abundant; $\mathrm{D}=$ dominant; $\mathrm{P}=$ poor; $\mathrm{F}=$ fair; $\mathrm{M}=$ moderate; $\mathrm{G}=$ good; $\mathrm{E}=$ excellent; ? = questionably present $;$. = not present. 